1 Characterization of the Endogenous DAF-12 Ligand and Its Use as an Anthelmintic Agent

\title{
in Strongyloides stercoralis
}

3

4 Zhu Wang ${ }^{1}$, Mi Cheong Cheong ${ }^{1}$, Jet Tsien ${ }^{2}$, Heping Deng ${ }^{2}$, Tian Qin ${ }^{2}$, Jonathan D. C.

5 Stoltzfus ${ }^{3}$, Tegegn G. Jaleta ${ }^{4}$, Xinshe Li ${ }^{4}$, James B. Lok ${ }^{4}$, Steven A. Kliewer ${ }^{1,5, *}$, and David J.

6 Mangelsdorf ${ }^{1,6, *}$

7

$8{ }^{1}$ Department of Pharmacology, University of Texas Southwestern Medical Center, Dallas, TX 9 75390, USA

$10{ }^{2}$ Department of Biochemistry, University of Texas Southwestern Medical Center, Dallas, TX

11 75390, USA

$12{ }^{3}$ Department of Biology, Millersville University of Pennsylvania, Millersville, PA 17551, USA

$13{ }^{4}$ Department of Pathobiology, School of Veterinary Medicine, University of Pennsylvania,

14 Philadelphia, PA 19104, USA

$15{ }^{5}$ Department of Molecular Biology, University of Texas Southwestern Medical Center, Dallas, 16 TX 75390, USA

$17{ }^{6}$ Howard Hughes Medical Institute, University of Texas Southwestern Medical Center, Dallas, 18 TX 75390, USA

19 *Correspondence: steven.kliewer@utsouthwestern.edu (S.A.K.),

20 davo.mango@utsouthwestern.edu (D.J.M.)

21 


\section{ABSTRACT}

23 A prevalent feature of Strongyloides stercoralis is a life-long and potentially lethal infection that

24 is due to the nematode parasite's ability to autoinfect and, thereby, self-replicate within its host.

25 Here, we investigated the role of the parasite's nuclear receptor, Ss-DAF-12, in governing infection.

26 We identified $\Delta 7$-DA as the endogenous Ss-DAF-12 ligand and elucidated the hormone's

27 biosynthetic pathway. Genetic loss of function of the ligand's rate-limiting enzyme demonstrated

28 that $\Delta 7$-DA synthesis is necessary for parasite reproduction, whereas its absence is required for

29 development of infectious larvae. Availability of the ligand permits $S s-D A F-12$ to function as an

30 on/off switch governing autoinfection, making it vulnerable to therapeutic intervention. In a

31 preclinical model of hyperinfection, pharmacologic activation of DAF-12 suppressed

32 autoinfection and markedly reduced lethality. Moreover, when $\Delta 7-\mathrm{DA}$ was administered with

33 ivermectin, the current but limited drug of choice for treating strongyloidiasis, the combinatorial

34 effects of the two drugs resulted in a near cure of the disease. 


\section{INTRODUCTION}

Strongyloidiasis is a neglected tropical disease caused by the nematode parasite, Strongyloides stercoralis. It is estimated to infect $\sim 600$ million people worldwide and is endemic in Africa, Asia, Latin America, and parts of the Caribbean, southern United States and Europe (Buonfrate et al., 2020). Because of the unique nature of its lifecycle, S. stercoralis infections are often life-long and up to $2.5 \%$ of these infections will progress to a hyperinfection syndrome that has a 90\% mortality rate if untreated (Milder et al., 1981). Notably, such hyperinfections are often caused by administering glucocorticoids to otherwise asymptomatic patients (Milder et al., 1981). The lifecycle of S. stercoralis is atypical of most soil-transmitted helminths (Krolewiecki et al., 2013; Lok, 2007; Page et al., 2018) (Figure 1). Infective third stage larvae (L3i) are developmentally quiescent and reside in fecal-contaminated soil until they contact the host and penetrate the skin, a process that reactivates their development as L3+ larvae. In the host, the activated L3+ immediately begin feeding, and typically migrate to the lungs, enter the alimentary canal, and transit to the intestine where the stage 4 larvae (L4) mature into parasitic (P)-adult

50 females (there are no parasitic males). The P-adult females reproduce asexually and generate rhabditiform, non-infectious stage 1 larvae (L1) progeny that can enter two alternate lifecycle

52 routes, one in the external environment and one in the host. Post-parasitic L1 (PP-L1) enter the external environment by leaving the host with the feces. Under conditions that mimic the host intestine (e.g., $37^{\circ} \mathrm{C}$ ), most PP-L1 develop as females directly into infectious filariform L3i

55 (Albarqi et al., 2016). However, under favorable conditions (e.g., $22^{\circ} \mathrm{C}$, high humidity) the majority of PP-L1 undergo a developmental switch and become free-living (FL) male and female

57 larvae that eventually mature into FL-adults, which are morphologically distinct from parasitic 
58 females and feed on soil microbes. The FL-adults mate and produce post-free-living (PFL) larvae that are exclusively female and now definitively committed to become infective L3i larvae. In addition to the conditions that produce L3i outside of the host, S. stercoralis has the

61 ability to remain in the host and undergo continuous autoinfection (Figure 1). This is due to the

62 presence of L1 larvae that develop directly into autoinfective third-stage larvae (L3a) within the

63 intestine. Similar to their L3i counterparts, L3a infect the host, in this case by penetrating the

64 intestinal wall and entering parenteral tissues as activated L3+ to complete the parasitic lifecycle. Autoinfection is unique to $S$. stercoralis and allows the parasite to persist in the host for decades as a latent, often asymptomatic infection. However, under certain host conditions such as

67 immunosuppression by glucocorticoids, the autoinfection spirals out of control, dramatically 68 increasing the parasite burden. The resulting hyperinfection leads to a breakdown of the intestinal mucosal barrier and dissemination of invasive larvae throughout the body accompanied by bacterial infection leading to sepsis that is responsible for the high mortality. The current treatment of choice is the nematode-selective chloride channel activator ivermectin, which is effective at controlling acute infections, but is limited by its inability to eradicate the persistent autoinfective

73 larvae (Krolewiecki et al., 2013; Repetto et al., 2018). Perhaps for this reason, the efficacy of

74 ivermectin in preventing fatality due to the hyperinfection and disseminated forms of the disease 75 is as low as 50\% (Buonfrate et al., 2013), and cases of ivermectin resistance are now being reported 76 in other nematode parasites (Prichard, 2007). The current increased prevalence of strongyloidiasis

77 has stimulated efforts to include increased dosing of ivermectin through mass drug administration 78 (Bisoffi et al., 2013), which in the long-term could accelerate ivermectin resistance in S. stercoralis.

79 Of further immediate concern, the risk of hyperinfection has risen dramatically with the use of 80 glucocorticoids to treat inflammatory diseases, particularly SARS-CoV-2 (Moloo, 2020). For 
81 these reasons, there is an urgent need to develop new therapeutic strategies for treating

82 strongyloidiasis (Krolewiecki et al., 2013; Moloo, 2020).

The lifecycle of $S$. stercoralis has similarities to the free-living nematode, C. elegans. The developmentally arrested L3i stage of $S$. stercoralis is analogous to the L3 dauer (d) stage of $C$. elegans. In C. elegans, L3d development is governed by DAF-12 (Antebi et al., 1998; Antebi et al., 2000), a nematode-specific nuclear receptor that is conserved in parasitic species (Ayoade et al., 2020; Long et al., 2020; Ma et al., 2019; Motola et al., 2006; Ogawa et al., 2009; Wang et al., 2017; Wang et al., 2009). In favorable environments, developing larvae synthesize the endogenous C. elegans (Ce)-DAF-12 ligands called dafachronic acids (Mahanti et al., 2014; Motola et al., 2006). The most potent and abundant of these ligands is $\Delta 7$-dafachronic acid ( $\Delta 7$-DA), which induces a transcriptional program that governs metabolism and reproductive growth to mature adults (Bethke et al., 2009; Hammell et al., 2009; Wang et al., 2015). The final and rate-limiting step of $\Delta 7-\mathrm{DA}$ synthesis is catalyzed by DAF-9, a cytochrome P450 (Gerisch \& Antebi, 2004; Jia et al., 2002; Motola et al., 2006). In unfavorable environments, $\Delta 7-\mathrm{DA}$ is not produced and unliganded $C e$-DAF-12 functions as a transcriptional repressor that arrests growth as L3d larvae in a process known as dauer diapause. When favorable conditions return, $\Delta 7-\mathrm{DA}$ synthesis resumes, DAF-12 is transcriptionally active, and worms exit L3 dauer and continue reproductive development. Our previous work demonstrated a similar requirement for the orthologous $S$. stercoralis (Ss)-DAF-12 receptor in governing L3i (Albarqi et al., 2016; Wang et al., 2009). Although the endogenous Ss-DAF-12 ligand was unknown, we demonstrated that pharmacologic administration of $\triangle 7$-DA to PP-L1 or PFL-L1 larvae commits them to free-living reproductive development, even under conditions that would normally commit them to become L3i (Albarqi et al., 2016; Wang et al., 2009), and institutes a transcriptomic profile in L3i similar to L3+ (Stoltzfus 
et al., 2014). In addition, knockout of Ss-DAF-12 prevents the formation of L3i larvae (Cheong et al., 2021), and in a mouse model of hyperinfection, exogenous $\Delta 7$-DA treatment lowers the worm burden in the intestine (Patton et al., 2018).

Despite these findings, an understanding of the DAF-12 signaling pathway during parasitism and importantly whether targeting the $S s$-DAF-12 receptor is a viable, curative strategy for treating strongylodiasis is lacking. In this report, we identified the endogenous $S s$-DAF-12 ligand, characterized its biosynthetic pathway, and demonstrated how its regulation governs the parasite's lifecycle. Finally, using a relevant pre-clinical animal model, we show that pharmacological activation of Ss-DAF-12 overcomes the deficiencies of ivermectin, and that when used in combination with ivermectin, provides a combinatorial therapeutic response that may be curative.

\section{RESULTS}

\section{$\Delta 7-D A$ is the endogenous ligand for DAF-12 in $S$. stercoralis}

Our previous work had demonstrated $\Delta 7$-DA is able to bind and activate $S s$-DAF-12 (Wang

et al., 2009). However, whether $\Delta 7$-DA or a related molecule is the endogenous S. stercoralis ligand is unknown. Indeed, the finding that the ligand binding pocket of Ss-DAF12 is divergent from that of $C e$-DAF-12 (Wang et al., 2009) and the observation that S. stercoralis lacks an obvious ortholog of DAF-9 (the only known DA-synthesizing enzyme found in C. elegans) suggested that the Ss-DAF-12 ligand would likewise be divergent. To determine the identity of $S$. stercoralis DAF-12 ligands, we employed an unbiased, activity-based biochemical purification strategy (Figure 2-figure supplement 1). In a control experiment using a DA-deficient strain of $C$. 
elegans supplemented with a known quantity of DA, we demonstrated this strategy effectively recovered $70 \%$ of the ligand (Figure 2-figure supplement $1 \mathrm{~B}$ ). Following this strategy, we fractionated lipids extracted from the FL-L3 larvae of S. stercoralis, which are analogous to the L3 stage of C. elegans where DA levels are highest (Li et al., 2013; Motola et al., 2006). Among the 70 HPLC fractions, $S s$-DAF-12 ligand activity was detected only in fraction 23 in a cell-based reporter assay and this activity was dose-dependent (Figure 2A). Remarkably, fraction 23 coeluted with dafachronic acids (Figure 2-figure supplement 1B), suggesting that the ligand is a related molecule.

To test whether the identified ligand activity is a dafachronic acid, we utilized a method incorporating ultra-performance liquid chromatography coupled with mass spectrometry (UPLCMS) that specifically detects all known DA ligands (e.g., $\Delta 4-\mathrm{DA}, \Delta 7-\mathrm{DA}$, and $\Delta 1,7-\mathrm{DA}$ ). Surprisingly, comparing the UPLC-MS data to DA standards (Table 1) revealed the presence of $\Delta 7$-DA (Figure 2B) but no other congeners, such as $\Delta 4$-DA and $\Delta 1,7-\mathrm{DA}$ (Figure 2-figure supplement 2). The predicted endogenous concentration of $\Delta 7$-DA in $S$. stercoralis FL-L3 is $\sim 200$ $\mathrm{nM}$, which is well above the concentration needed to fully activate $S s$-DAF-12 (Figure 2B, inset). Taken together, these data demonstrate that $\Delta 7-\mathrm{DA}$ is present in $S$. stercoralis and acts as an endogenous DAF-12 ligand.

\section{$\Delta 7-D A$ levels correlate with reproductive development in $S$. stercoralis lifecycle}

In C. elegans, DAF-12 ligands promote reproductive development (Bethke et al., 2009; Hammell et al., 2009; Motola et al., 2006) and adulthood longevity (Gerisch et al., 2007; Yamawaki et al., 2010). To examine where in the $S$. stercoralis lifecycle $\Delta 7-\mathrm{DA}$ is present, lipid extracts from each developmental stage were analyzed by UPLC-MS (Figure 3). In the postparasitic environment under conditions that promote free-living development, $\Delta 7$-DA was present 
in FL-L1/L2 and was significantly more abundant in the FL-L3 stage as the larvae progress toward reproductive maturity (Figure 3A-C). Similarly, $\Delta 7$-DA was detected in L3+ that have just infected the host and begun their reproductive development (Figure 3A-C). These findings are consistent with what is observed in C. elegans undergoing normal reproductive development (Li et al., 2013; Motola et al., 2006). A unique aspect of $S$. stercoralis is that after one free-living generation outside of the host, PFL larvae are fated to growth arrest at L3i. In concordance with the hypothesis that developmental arrest is due to the absence of a DAF-12 ligand, $\triangle 7$-DA was undetectable in FLadults, PFL-L1, and L3i larvae (Figure 3A-C).

The intestinal (Int)-L1-L3a larvae that were the direct progeny of parasitic adults in hyperinfected gerbils also had virtually no $\Delta 7$-DA (Figure 3A-C). These larvae that remain within the intestine are committed to become infectious as L3a, similar to their post-free living counterparts that become L3i (Lok, 2007; Viney \& Lok, 2015). By comparison, in the external post-parasitic environment under temperature conditions that mimic the host intestine, the PP-L1 are also fated to become infectious as L3i (Figure 3C) (Albarqi et al., 2016). The absence of $\Delta 7$ DA in these two stages (L3i and L3a) is similar to the developmental diapause observed in $C$. elegans, in which larvae arrest at the L3d stage in the absence of the DAF-12 ligand (Motola et al., 2006). Taken together, these results indicate that liganded DAF-12 in S. stercoralis is required for reproductive development, while unliganded DAF-12 is requisite for production of infectious L3i and L3a larvae.

Another intriguing finding was that the highest concentration of $\Delta 7-\mathrm{DA}$ occurred in parasitic adults, as opposed to the essentially undetectable levels in free-living adults (Figure 3B). This difference in $\Delta 7$-DA levels correlated with the markedly different lifespans of the two adult populations. Parasitic adults live for up to a year or more in the host, whereas free-living adults 
172 survive for only a few days in soil (Lok, 2007; Viney \& Lok, 2015). Given that $\Delta 7$-DA is known

173 to extend lifespan of adult $C$. elegans (Gerisch et al., 2007; Yamawaki et al., 2010), this finding

174 suggests that $\triangle 7$-DA may play a similar role in extending the lifespan of parasitic adults in $S$.

175 stercoralis.

176

177

178

179

180

181

182

183

184

185

186

187

188

189

190

191

192

193

194

\section{Identification of the $\Delta 7-\mathrm{DA}$ biosynthetic pathway in $S$. stercoralis}

In C. elegans, DAF-12 ligands are synthesized from dietary cholesterol by a cascade of enzymes including a Rieske oxygenase (DAF-36), a short-chain hydroxysteroid dehydrogenase (DHS-16), and a cytochrome P450 (CYP; DAF-9) that catalyzes the final and rate-limiting step in dafachronic acid synthesis (Figure 4A) (Motola et al., 2006; Rottiers et al., 2006; Wollam et al., 2012; Wollam et al., 2011). Bioinformatic analysis of the S. stercoralis genome revealed single homologs for both DAF-36 and DHS-16 (Stoltzfus et al., 2012), which share sequence identity with their C. elegans counterparts. In contrast, there are 26 CYPs in S. stercoralis, but none of these share predictive sequence identity with DAF-9 ( 36\% at the best) (Stoltzfus et al., 2012). The lack of an obvious DAF-9 ortholog suggested the possibility that $\Delta 7-\mathrm{DA}$ might be acquired exogenously by $S$. stercoralis. To rule out this possibility, we first determined whether $S$. stercoralis synthesize $\Delta 7$-DA from dietary cholesterol in vivo. Free-living PP-L1 were cultured on a diet where the natural cholesterol isotope (largely ${ }^{12} \mathrm{C}$-cholesterol) was supplemented with ${ }^{13} \mathrm{C}$-labeled cholesterol. After 24 hours, we detected significant levels of ${ }^{13} \mathrm{C}$-labeled $\Delta 7$-DA in the FL-L3 (Figure 4B). These results demonstrated that $\Delta 7-\mathrm{DA}$ is generated from dietary cholesterol in S. stercoralis and that the parasite has all of the necessary enzymatic machinery to perform the synthesis.

To characterize the biosynthetic enzymes, we employed a Sf9 cell expression system that we used to identify the $C$. elegans counterparts of these proteins (Motola et al., 2006; Rottiers et 
al., 2006; Wollam et al., 2012; Wollam et al., 2011). To avoid potential interference from any endogenous substrates in $\mathrm{Sf} 9$ cells, we utilized ${ }^{2} \mathrm{H}$-isotope-labeled substrates to assay the activity of the candidate S. stercoralis orthologs, Ss-DAF-36 (SSTP_0000037900) and Ss-SCDH-16 (SSTP_0001031100, the homolog of Ce-DHS-16). Sf9 microsomes expressing Ss-DAF-36 readily converted cholesterol to 7-dehydrocholesterol (Figure 4C) with an efficacy comparable to the $C$. elegans enzyme (Figure 4D). Similarly, Ss-SCDH-16 catalyzed the conversion of lathosterol to lathosterone like Ce-DHS-16 (Figure 4E, F). These findings demonstrated that S. stercoralis has functional orthologs of DAF-36 and DHS-16.

The inability to predict the parasite's DAF-9 ortholog from our bioinformatic analysis prompted us to perform an unbiased screen to identify the enzyme from among all 26 S. stercoralis CYPs. Each of the 26 CYP enzymes was expressed in Sf9 cells (Figure 4-figure supplement 1) and assayed by UPLC-MS for their ability to synthesize $\Delta 7$-DA from lathosterone. Unambiguously, only one of the 26 CYPs, Ss-CYP22a9 (SSTP_0001032100) synthesized $\Delta 7$-DA at levels comparable to Ce-DAF-9 (Figure 4G). These results demonstrated that $S s-\mathrm{CYP} 22 \mathrm{a} 9$ is the DAF9 isoenzyme in $S$. stercoralis.

\section{$\Delta 7-D A$ synthesis is required for reproductive development in $S$. stercoralis}

In C. elegans, favorable environmental cues induce DAF-9 expression and DAF-12 ligand synthesis through a cGMP signaling pathway, leading to reproductive development $(\mathrm{Hu}, 2007)$. Likewise in S. stercoralis, favorable host conditions act through a similar pathway to stimulate feeding, a sign of reactivated development in L3i (Stoltzfus et al., 2014). To study the role of $\Delta 7$ DA synthesis in parasite reproductive development, we first asked whether $\Delta 7$-DA is made in response to activation of the cGMP pathway in L3i. To that end, we treated L3i with 8-Br-cGMP, which mimics the presence of the host environment by activating the cGMP signaling pathway, 
218 and then tested endogenous $\Delta 7$-DA levels in the L3i larvae. In the presence of 8-Br-cGMP, we

219 found that endogenous $\Delta 7$-DA was increased progressively over a 3-day period to levels $(\sim 100$ $\mathrm{nM}$ ) that would saturate $S s$-DAF-12 occupancy (Figure 5A). Furthermore, this stimulation of $\Delta 7$ -

221 DA synthesis correlated exactly with the expression of Ss-cyp22a9 (Figure 5-figure supplement

222 1A) and was abolished by co-treatment with ketoconazole, a broad-spectrum CYP inhibitor

223 (Figure 5A). These results highlight the importance of $\Delta 7$-DA synthesis in the reactivation of L3i

224 larval development.

225 We next tested the requirement of $S s-C Y P 22 a 9$ for $\Delta 7-\mathrm{DA}$ synthesis and parasite 226 development in vivo. For these experiments, a CRISPR-mediated, homology-directed gene 227 knockout strategy was used to insert a GFP expression cassette into the Ss-cyp22a9 gene locus 228 through homologous repair (Figure 5-figure supplement 1B, C). This strategy allowed us to enrich 229 for Ss-cyp22a9 knockout worms by selecting GFP-positive L3i. Single worm genotyping 230 confirmed the presence of at least one disrupted Ss-cyp22a9 allele in $>60 \%$ of the GPF-positive L3i (Figure 5B). To evaluate the phenotype due to loss of Ss-CYP22a9, we assayed cGMP-induced 232 feeding behavior in $\mathrm{L} 3 \mathrm{i}$, which as noted above is an early marker of the transition to reproductive 233 development (Lok, 2007; Stoltzfus et al., 2014; Viney \& Lok, 2015). In both wildtype larvae and 234 CRISPR-control larvae in which a gene unrelated to development (Ss_unc-22) was targeted, 8-Br235 cGMP strongly induced the feeding behavior as expected (Figure 5C). In contrast, in Ss-cyp22a9 236 knockout larvae, cGMP-induced feeding behavior was severely impaired (Figure 5C). The residual 237 cGMP-induced response is likely due to the presence of an incomplete penetrance of the knockout 238 in all worms. The loss of this feeding behavior in Ss-cyp22a9-deficient larvae was rescued 239 completely by treatment with exogenous $\Delta 7-\mathrm{DA}$, further demonstrating the importance of DAF24012 activation as a requirement for parasite development (Figure 5C). To confirm the enzymatic 
241 function of Ss-CYP22a9, we also analyzed the production of $\Delta 7-\mathrm{DA}$ in $S s-c y p 22 a 9$ knockout L3i

242 larvae. For this experiment, we targeted the $S s$-cyp22a9 gene using non-homologous end joining

243 CRISPR with the same sgRNA as in Figure 5C and Figure 5-figure supplement 1C. This CRISPR

244 method permitted the generation of larger numbers of Ss-cyp22a9 knockouts, which were

245 necessary to detect endogenous $\Delta 7$-DA levels. Consistent with the results of the CYP inhibitor,

246 disrupting the Ss-cyp22a9 gene abolished the $\Delta 7-\mathrm{DA}$ induction by cGMP (Figure 5D). Taken

247 together, these results demonstrated the crucial role of $S s-C Y P 22 \mathrm{a} 9$ in $\Delta 7-\mathrm{DA}$ synthesis and

248 highlight the importance of $\Delta 7$-DA synthesis in the development of infectious larvae in $S$.

249 stercoralis.

250

251

252

253

254

255

256

257

258

259

260

261

262

\section{$\Delta 7-D A$ suppresses parasitic burden in uncomplicated strongyloidiasis}

In S. stercoralis, uncomplicated infections are frequently asymptomatic but can last for years due to the persistence of L3a worms that escape the host immune system and continually reinfect the host at a low level. However, when the host is immune suppressed and the intestinal barrier is compromised, autoinfection accelerates out of control as more and more L3a worms invade the host, leading to a lethal hyperinfection. The results above suggested that targeting the DAF-12 pathway might provide a novel therapeutic opportunity for treating this disease. This strategy is based on the idea that like their L3i counterparts in the environment, the lack of $\Delta 7$-DA is required for intestinal larvae to become infectious L3a (Figure 3C). Thus, we postulated that pharmacological activation of DAF-12 in intestinal larvae should block autoinfection by preventing the formation of filariform L3a larvae as it does with L3i in the environment (Albarqi et al., 2016; Wang et al., 2009). We first asked whether $\Delta 7$-DA can successfully treat the uncomplicated, latent form of the disease. To that end, we employed a well-established gerbil 
model that mimics both uncomplicated and hyperinfective strongyloidiasis found in humans (Kerlin et al., 1995; Nolan et al., 1993).

For the uncomplicated model, we infected gerbils with 1000 L3i and after 21 days began administering vehicle or $\Delta 7$-DA orally in their drinking water, which delivers the compound directly into the gut where the intestinal larvae reside. We then monitored the parasite burden of the intestine by counting the number of fecal larvae. Compared to vehicle, treatment with $\Delta 7-\mathrm{DA}$ for 14 days dramatically reduced fecal larval output by $\sim 90 \%$ (312 \pm 103 larvae per gram feces for vehicle versus $32 \pm 6$ for DA, $q<0.03$; Figure $6 \mathrm{~A}$ ). In contrast, the numbers of parasitic adults, which produce intestinal larvae, were not significantly changed by $\Delta 7$-DA treatment (Figure $6 \mathrm{~B}$ ). Because of the time-frame of this experiment and the fact that autoinfection is minimal in the uncomplicated model (Nolan et al., 1993), reinfection of the host by L3a larvae is negligible, demonstrating that these adult parasites were derived from the L3i of the initial infection. This explains why the numbers of adults (in contrast to the intestinal larvae) were not substantially reduced by $\Delta 7$-DA treatment. These results are also consistent with our observation that intestinal larvae lack $\Delta 7$-DA (Figure 3), which is essential for L3a development, thereby supporting the hypothesis that $\Delta 7$-DA specifically targets the intestinal larval stages of the lifecycle. We conclude that $\Delta 7$-DA treatment impedes the development of intestinal larvae and thus blocks transmission in uncomplicated infections where conventional treatments are only partially effective.

\section{$\Delta 7-D A$ improves survival of a lethal strongyloidiasis hyperinfection}

Based on the finding that $\triangle 7$-DA disrupts intestinal larval development (Figure 6A), we next asked whether the DAF-12 ligand would be effective at treating the lethal form of strongyloidiasis. For this experiment, beginning at the time of infection with L3i, gerbils were administered the glucocorticoid, methylprednisolone, on a weekly basis (Nolan et al., 1993). 
Similar to what is observed in immunocompromised humans, this results in the rapid progression of disease to a disseminated hyperinfection that is lethal to all of the animals, with $>90 \%$ of them dying within 40 days (Figure 7A, black line). In contrast, when a matched group of these animals was treated with $\Delta 7$-DA starting at 10 days after the hyperinfection was initiated, there was an impressive $70 \%$ survival rate that lasted for at least 90 days at which point we arbitrarily terminated

302 Figure 7D). This difference is because in a hyperinfection the parasitic adults are largely derived

303 from the expanding L3a population, whereas in an uncomplicated infection the number of adults 304 that were present matured directly from the L3i larvae used for the initial infection. Overall, these 305 findings demonstrated that $\Delta 7-\mathrm{DA}$ treatment is effective in treating a lethal S. stercoralis 306 hyperinfection. 
As the current front-line drug for treating strongyloidiasis, ivermectin is effective at removing adult $S$. stercoralis parasites (Krolewiecki et al., 2013; Repetto et al., 2018). However, in hyperinfected patients ivermectin treatment is only $50-60 \%$ effective at preventing death

311 (Buonfrate et al., 2013). This high mortality is due to the relatively low efficacy of ivermectin at

312 killing the persistent autoinfective L3a larvae. Given that $\Delta 7$-DA specifically targets the L3a

313 population, we hypothesized that the combination of $\Delta 7-\mathrm{DA}$ and ivermectin would act

314 cooperatively to treat a strongyloidiasis hyperinfection. To test this, we first established an 315 ivermectin treatment model in hyperinfected gerbils that mimics the efficacy observed in humans

316 (Figure 7-figure supplement 1). In this model, $300 \mu \mathrm{g} / \mathrm{kg}$ ivermectin delivered i.p. acutely cleared

317 fecal larva output in $\sim 40 \%$ of the hyperinfected gerbils within 7 days, resulting in $\sim 25 \%$ survival

318 rate for at least 90 days (Figure 7A, red line). As expected, ivermectin was as effective as $\Delta 7$-DA

319 in removing adult parasites from animals undergoing hyperinfection (Figure 7D), but in contrast to $\Delta 7$-DA, ivermectin was ineffective at decreasing either the intestinal or L3+ larval populations

321 (Figure 7B, C). Next, we treated hyperinfected gerbils with both $\Delta 7$-DA and ivermectin. We found 322 that the combination of both agents improved the survival rate significantly over either agent alone $323(>75 \%$, Figure 7A) and reduced the parasite burden of all life stages (Figure 7B-D). Remarkably, 324 the combination of $\Delta 7-\mathrm{DA}$ and ivermectin completely eliminated the parasites from all the animals 325 that survived, essentially curing them of the disease (Figure 7E). In contrast, when treated with 326 ivermectin or $\Delta 7$-DA alone, $\leq 10 \%$ were parasite-free even among those animals that survived 327 (Figure 7E). Taken together, these results demonstrated that $\Delta 7-\mathrm{DA}$ and ivermectin act 328 synergistically in successfully eliminating $S$. stercoralis by targeting complementary stages of the 329 lifecycle (Figure 8). 


\section{DISCUSSION}

The worldwide prevalence of $S$. stercoralis infections and the increased risk of deadly hyperinfection due to the broad use of glucocorticoids to treat inflammatory diseases underscores the urgent need for new therapeutic approaches to treat strongyloidiasis (Buonfrate et al., 2013; Repetto et al., 2018). The difficulty in treating the disease arises from a persistent autoinfection in which L3a are produced and continuously re-infect the host. Consequently, the parasite burden can become uncontrollably large when the immune system is compromised, leading to frequent lethality. Our previous studies have suggested one potential target of interest is the nuclear receptor, DAF-12 (Patton et al., 2018; Wang et al., 2017; Wang et al., 2009), which is required for the transition into and out of the L3i stage of the parasite's lifecycle (Cheong et al., 2021). Here, we addressed two key unanswered questions: what is the nature and role of the Ss-DAF-12 ligand,

342 and is targeting the Ss-DAF-12 pathway a viable therapeutic strategy? In this report, we identified $\Delta 7-\mathrm{DA}$ as the endogenous ligand of Ss-DAF-12 and showed that the regulation of its biosynthesis

345 Similar to the free-living nematode, C. elegans, we found the presence of $\Delta 7-\mathrm{DA}$ is a requisite

346 feature for $S$. stercoralis free-living and parasitic larvae to mature into reproductive adults. In 347 contrast, we discovered $\triangle 7-\mathrm{DA}$ is absent during the formation of L3a. Thus, like the analogous 348 development of L3i in the environment, unliganded DAF-12 appears to be required for L3a 349 formation in hosts. Taken together, these findings revealed a vulnerability in the life cycle of $S$. stercoralis and suggested two new therapeutic strategies: either blocking the synthesis of the ligand 
To that end, we showed that knocking out the $\Delta 7$-DA biosynthetic enzyme prevents the

parasite's autoinfection cycle in both uncomplicated and hyperinfection forms of strongyloidiasis.

Strikingly, when $\Delta 7$-DA was combined with the front-line medication, ivermectin, there was a surviving animals receiving both $\Delta 7$-DA and ivermectin, no parasites from any stage were detected anywhere in the body, suggesting that the co-treatment resulted in a complete cure.

\section{Advantages of DAF-12 as a Therapeutic Target}

The synergistic response of $\Delta 7-\mathrm{DA}$ and ivermectin highlights the unique potential of targeting the DAF-12 signaling pathway and overcoming the limitations of using ivermectin alone. Mortality rates in ivermectin-treated patients with hyperinfection range as high as $50 \%$ (Buonfrate et al., 2013). As an inhibitor of muscular function, ivermectin is highly effective at killing adults

367 and feeding larvae (Repetto et al., 2018), but less effective at killing the developmentally quiescent L3a larvae that continuously drive autoinfection. In contrast, $\Delta 7$-DA directly interferes with infectious L3a larval development by binding to unliganded DAF-12 and provoking an inappropriate developmental response inside the host that results in death of the larvae. In this way,

371 the two drugs complement each other by targeting different stages of the lifecycle (Figure 8).

372 Targeting the ligand binding properties of DAF-12 has other distinct advantages in that this nuclear

373 receptor is nematode-specific and mutants resistant to pharmacologic ligands are unlikely to 374 develop, since such mutants would be expected to disrupt the endogenous function of the receptor 375 and otherwise be lethal. 


\section{The Conundrum of $\Delta 7-\mathrm{DA}$ as the $S s-\mathrm{DAF}-12$ Ligand}

An intriguing aspect of this study was the finding that $\triangle 7-\mathrm{DA}$ is the endogenous ligand for Ss-DAF12. Although $\Delta 7$-DA was first identified as the endogenous DAF-12 ligand in C. elegans, its presence in $S$. stercoralis was unexpected. In comparison to its $C$. elegans homolog, the SsDAF-12 ligand binding domain shares only moderate sequence identity (42\%) and exhibits a distinct pharmacologic profile in response to $\Delta 7$-DA compared to other species (Wang et al., 2009). The sequences of other regulatory proteins in the Ss-DAF-12 pathway also have diverged. For example, the cytochrome P450 (Ss-CYP22a9) that catalyzes the synthesis of $\Delta 7$-DA (Figure 4) and the ligand-dependent coactivator that $S s$-DAF-12 requires for transactivation (Ss-DIP-1) (Cheong et al., 2021) are both unique to Strongyloides spp.

In spite of these differences, several lines of evidence indicate that $\Delta 7-\mathrm{DA}$ is the major, if not exclusive, DAF-12 ligand in S. stercoralis. First, no other ligand activities, including other types of dafachronic acids were detected. Second, the endogenous concentration of $\Delta 7-\mathrm{DA}$ in $S$. stercoralis $(\sim 200 \mathrm{nM})$ is sufficient to drive full activation of Ss-DAF-12, leaving other potential DAF-12 ligands redundant if they do exist. Finally, knocking out the daf- 9 homolog, Ss-cyp22a9, which is required to synthesize $\triangle 7$-DA, prevents DAF-12-dependent activity in vivo, and that activity is rescued completely by adding back the ligand. Interestingly, $\Delta 7-\mathrm{DA}$ has been shown to activate DAF-12 homologs in other species (Ayoade et al., 2020; Long et al., 2020; Ma et al., 2019;

Ogawa et al., 2009; Wang et al., 2009). Why the $\Delta 7$-DA hormone has been evolutionarily conserved, whereas other components of the pathway have not, remains unclear, but nevertheless highlights the importance of the ligand in governing nematode biology.

\section{Therapeutic Potential Beyond S. stercoralis}


The findings discussed above underscore the potential of developing agonists that would precociously activate unliganded $S S$-DAF-12 and kill L3a larvae, a therapy that would be propitious and specific to $S$. stercoralis. However, the observation that liganded DAF-12 also is required at a completely different but essential stage of the lifecycle to promote reproductive growth raises the possibility of also targeting DAF-12 with an antagonist. Given that DAF-12 has been found in all nematode species surveyed to date (Ayoade et al., 2020; Long et al., 2020; Ma approach for treating most, if not all, nematode parasitisms. (Albarqi et al., 2016) supports this strategy. we note that $\triangle 7$-DA may not be the best candidate for eventual clinical use. Typical of most endogenous steroid receptor ligands, $\Delta 7-\mathrm{DA}$ has a short half-life and relatively poor

417 pharmacokinetic properties in vivo (Patton et al., 2018). For this reason, in our study we administered $\Delta 7$-DA continuously in the drinking water over many days. Although overcoming

419 this limitation would be important for developing a suitable therapeutic modality, this is not an 
421 circumvented through the design of potent, long-lasting receptor agonists and antagonists (Chen,

422 2008; Zhao et al., 2019). Another limitation of this study is that we only tested single doses of $\Delta 7$ -

423 DA and ivermectin. However, we note that optimizing the dose regimens are likely to reveal even

424 greater cooperative effects. Finally, we have not definitively ruled out the existence of other

425 endogenous DAF-12 ligands. While the findings that $\triangle 7$-DA is the only ligand we detected and is

426 present only at the times when DAF-12 would be expected to be an activator are consistent with

427 the conclusion that $\Delta 7-\mathrm{DA}$ is the relevant ligand, it is feasible that other activities exist that were

428 not identified in our purification scheme.

\section{Conclusions}

In summary, in this work we identified the endogenous DAF-12 ligand in the human

431 parasitic nematode S. stercoralis, characterized the ligand's biosynthesis pathway, and

432 demonstrated that activating DAF-12 can overcome the limitation of the front-line drug,

433 ivermectin, and significantly improve the outcome of strongyloidiasis in a preclinical model of

434 hyperinfection. Our study further revealed the ligand's biosynthetic enzyme as a potential new

435 target that might also be exploited to treat this lethal parasitic disease. 


\section{FIGURES}

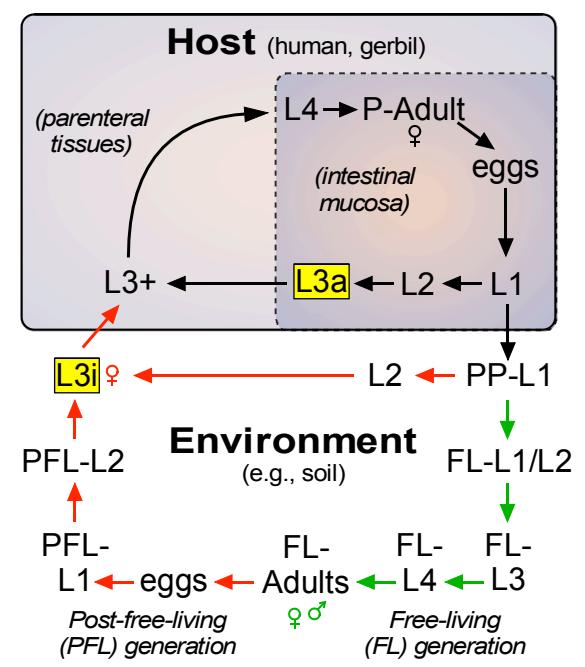

$438 \quad$ Figure 1. Life cycle of $\boldsymbol{S}$. stercoralis.

439 Life cycle of $S$. stercoralis. Similar to other nematodes, S. stercoralis hatch from eggs and undergo

440 four larval (L) molts to become adults. However, under certain conditions, the larvae arrest their

441 development as infectious third-stage larvae. The parasite has two infectious stages (highlighted

442 in yellow), one that exists in the environment as L3i and one that exists in the mucosa of the host

443 intestine as an autoinfective L3a. 

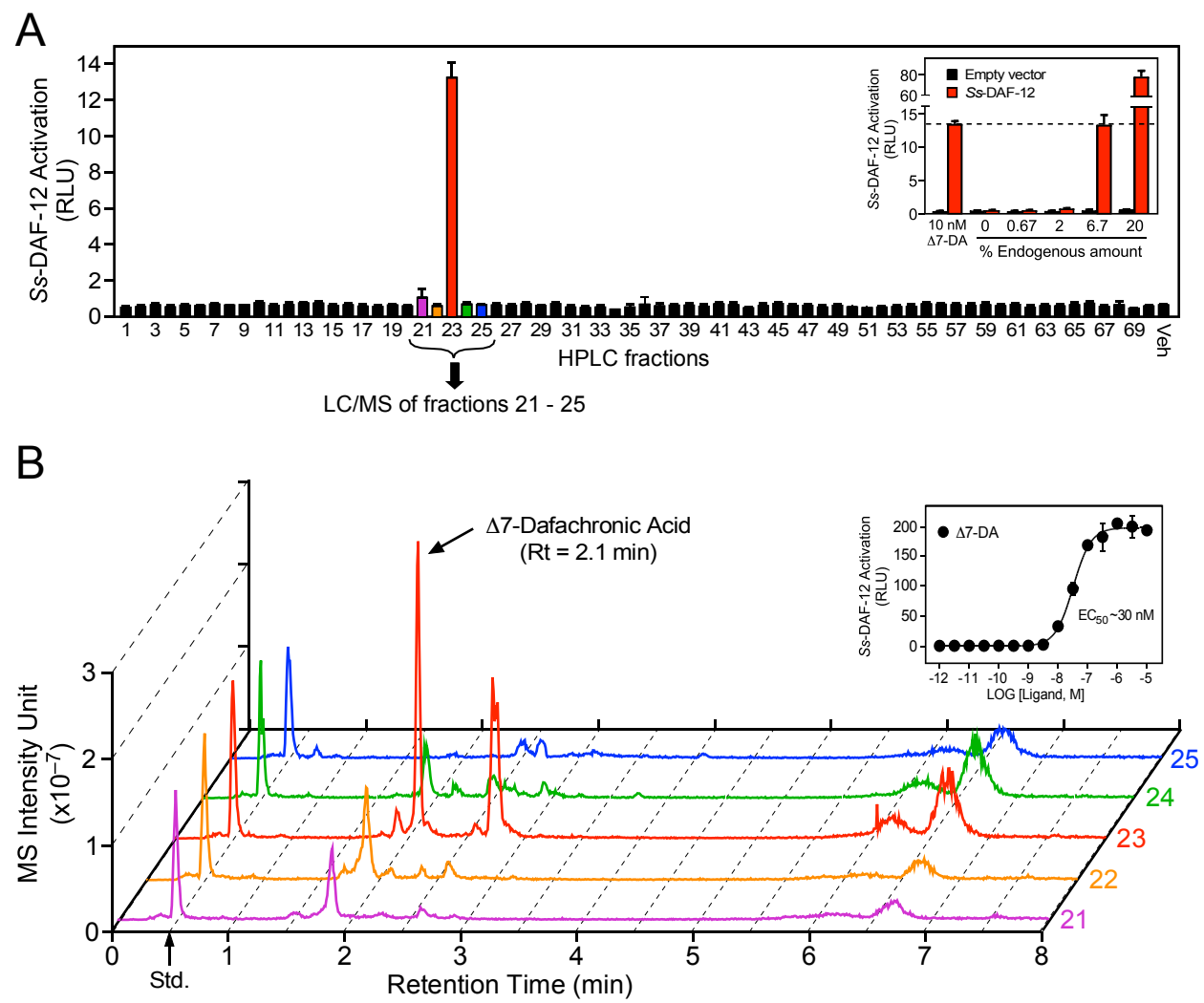

Figure 2. Identification of $\Delta 7$-dafachronic acid as the endogenous DAF-12 ligand in $S$. stercoralis.

(A) Purification of the endogenous $S$. stercoralis ligand for Ss-DAF-12. Lipids from post-parasitic resulting lipid fractions were then tested in a $S s-\mathrm{DAF}-12$ cell-based reporter assay. Inset: Dose response of the endogenous activity in fraction 23. RLU, relative luciferase units. Data are presented as the mean \pm s.d. of technical triplicates. assay. Data are presented as the mean \pm s.d. of technical triplicates and were repeated 3 times.

455 See also Figure 2-figure supplement 1 and 2. 


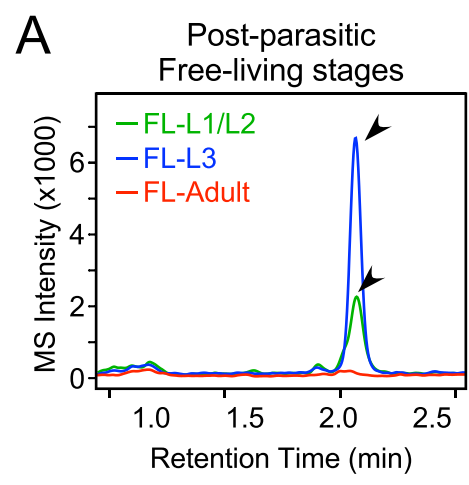

B

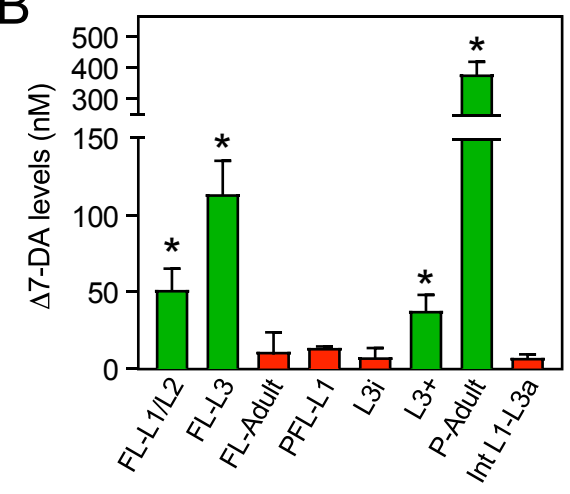

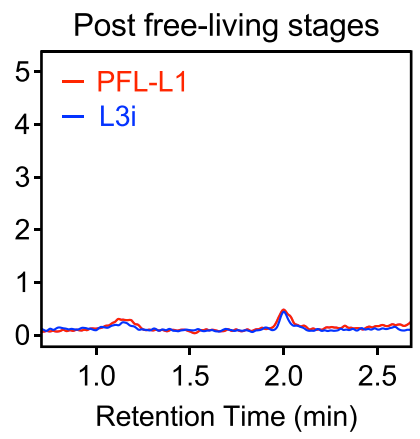
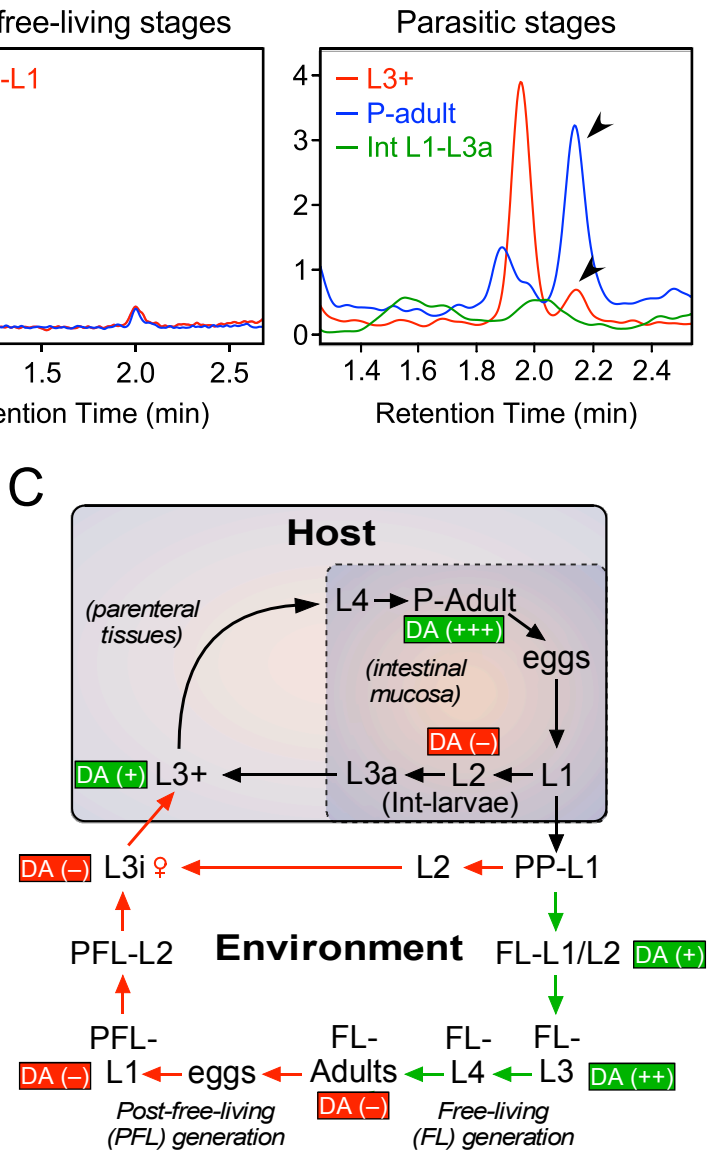

457 Figure 3. Profiling of $\Delta 7-\mathrm{DA}$ in developmental stages of $\boldsymbol{S}$. stercoralis.

458 (A) Detection of $\Delta 7$-DA during the lifecycle of $S$. stercoralis. Lipid extracts from the indicated stages of $S$. stercoralis were analyzed by derivatizing $\Delta 7$-DA to $\Delta 7$-DA-picolylamine, which then was detected by UPLC-MS in positive MRM mode with $\mathrm{m} / \mathrm{z}$ transition $505 \rightarrow 487$. Parasitic stages were recovered from hyperinfected gerbils. Arrowheads show $\Delta 7$-DA peaks. Note that in the extracts from $\mathrm{L} 3+$ larvae, the peak with the faster retention time $(1.95 \mathrm{~min})$ is an unknown metabolite that is only found at this stage.

(B) $\Delta 7$-DA levels were determined in the stages shown in (A) by comparison to a known standard. $\mathrm{n}=3 \pm$ s.d.; $*, \mathrm{p}<0.03$ by student t-test compared to L $3 \mathrm{i}$ larvae. 
466 (C) Schematic summary of $\Delta 7$-DA levels in the development stages of S. stercoralis. $\Delta 7$-DA is

467 absent in larvae developing to infectious stages (i.e., L3i and L3a) and is present in larvae

468 undergoing reproductive development. 
A

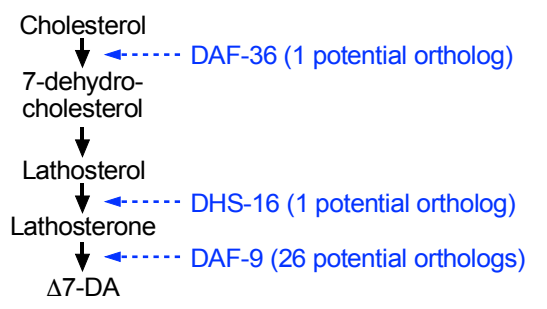

B
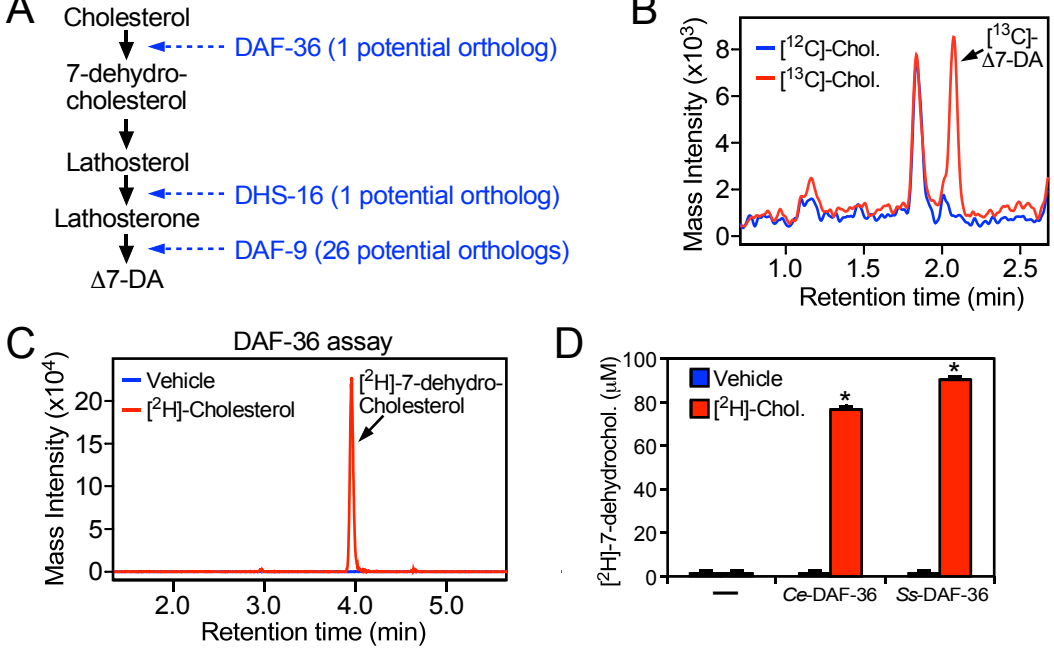

$\mathrm{D}$

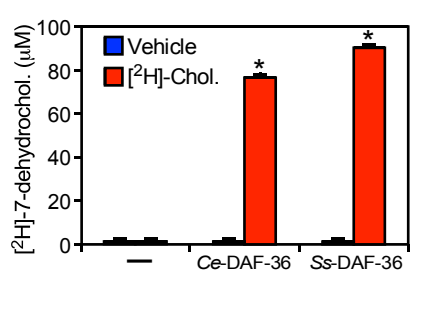

E

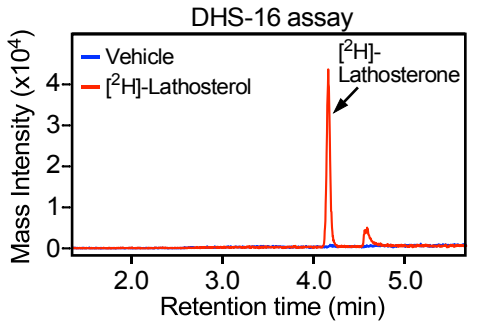

$\mathrm{F}$

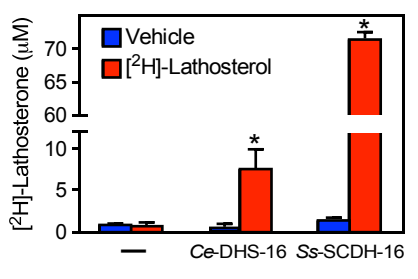

G

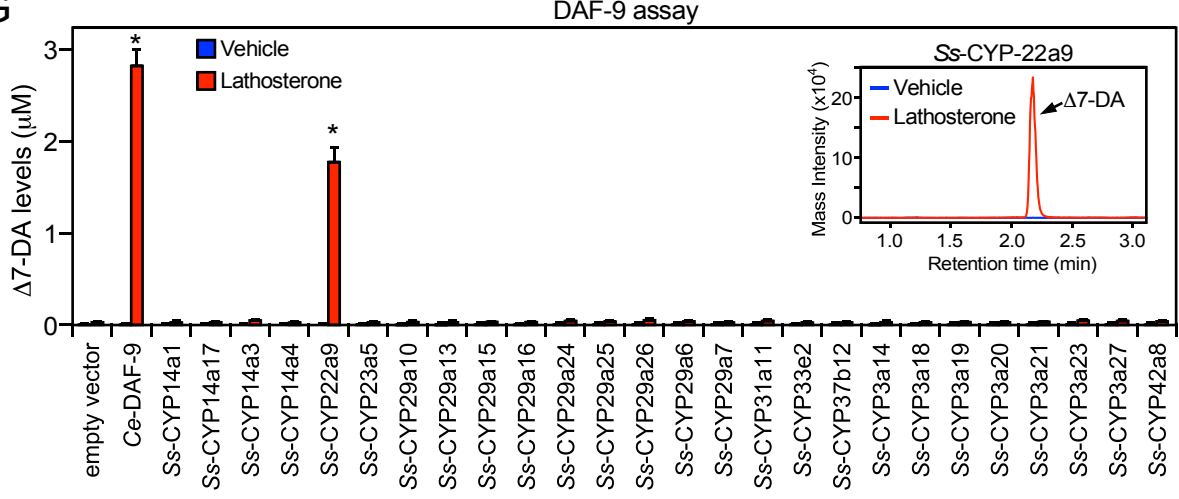

471 (A) Diagram of $\Delta 7$-DA biosynthetic pathway in C. elegans. In blue are the known C. elegans

472 enzymes followed in parentheses by the number of candidate orthologs found in S. stercoralis.

473 (B) $\Delta 7-D A$ is synthesized de novo in S. stercoralis. Extracts from post-parasitic L1 worms cultured

474 in the presence or absence of $\left[{ }^{13} \mathrm{C}\right]$-cholesterol were assayed by UPLC-MS for incorporation of $475 \quad\left[{ }^{13} \mathrm{C}\right]$ into $\Delta 7-\mathrm{DA}$. 
476 (C-D) $S s$-DAF-36 catalyzes the synthesis of 7-dehydrocholesterol in the first step of $\Delta 7$-DA

477 biosynthesis. Sf9 microsomes expressing Ss-DAF-36 were incubated with vehicle or $100 \mu \mathrm{M}\left[{ }^{2} \mathrm{H}\right]-$

478 cholesterol and assayed for the synthesis of $\left[{ }^{2} \mathrm{H}\right]-7$-dehydrocholesterol by UPLC-MS

479 chromatography (C), and the amount quantitated relative to that produced by the C. elegans

480 ortholog, Ce-DAF-36 (D).

481 (E-F) $S s-\mathrm{SCDH}-16$ catalyzes the of synthesis of lathosterone in the penultimate step of $\Delta 7-\mathrm{DA}$

482 biosynthesis. Sf9 microsomes expressing $S s-\mathrm{SCDH}-16$ were incubated with vehicle or $100 \mu \mathrm{M}$

$483\left[{ }^{2} \mathrm{H}\right]$-lathosterol and assayed for the synthesis of $\left[{ }^{2} \mathrm{H}\right]$-lathosterone by UPLC-MS chromatography

484 (E), and the amount quantitated relative to that produced by the C. elegans ortholog, Ce-DHS-16

$485(\mathrm{~F})$.

486 (G) Ss-CYP22a9 catalyzes the synthesis of $\Delta 7$-DA from lathosterone. Sf9 cells expressing one of

487 each of the $26 \mathrm{~S}$. stercoralis cytochrome P450 homologs were incubated with vehicle or $10 \mu \mathrm{M}$

488 lathosterone and assayed for the production of $\triangle 7$-DA by UPLC-MS as in Figure 2. Ce-DAF-9 is

489 shown as a positive control. Inset, chromatogram from the reaction with Ss-CYP22a9.

$490 \mathrm{n}=3 \pm$ s.d.; $*$, $<0.03$ by student t-test compared to vehicle.

491 See also Figure 4-figure supplement 1.

492 


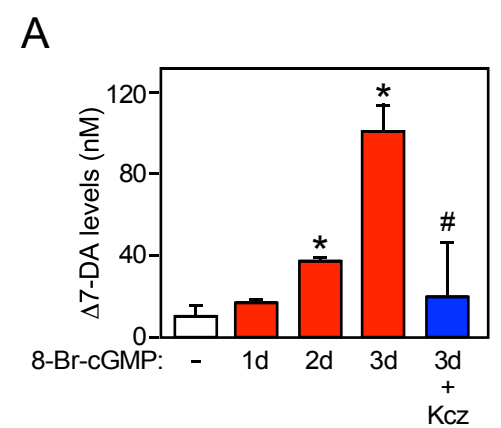

C

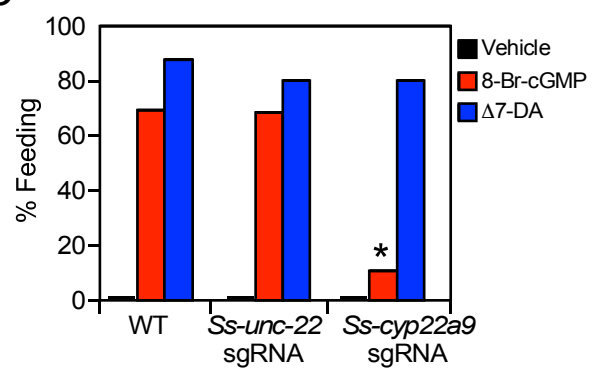

$\mathrm{B}$

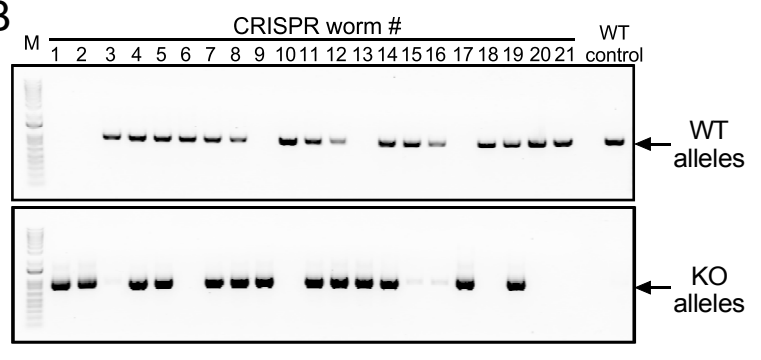

D

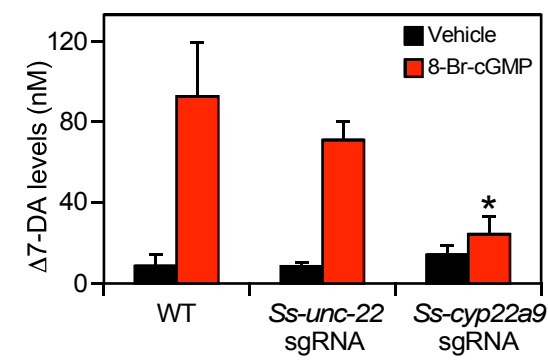

Figure 5. Ss-CYP22a9 is required for L3i activation and $\Delta 7-D A$ synthesis in $S$. stercoralis.

(A) Inhibition of cytochrome P450 activity blocks $\Delta 7-\mathrm{DA}$ synthesis in parasites. L3i (100 worms/group) were treated with $0.5 \mathrm{mM} \mathrm{8-Br-cGMP}$ in the presence or absence of $25 \mathrm{mM}$ ketoconazole $(\mathrm{Kcz}) . \mathrm{n}=3 \pm$ s.d.; $\mathrm{p}<0.03$ by t-test compared to vehicle $\left({ }^{*}\right)$ or $3 \mathrm{~d}$ treatment with 8-Br-cGMP (\#).

(B-C) $S s-C Y P 22 a 9$ is required for cGMP-induced L3i activation. The Ss-cyp22a9 gene was disrupted by CRISPR/Cas9-mediated, homology-directed repair as shown in Figure 5-figure supplement 1C. The resulting F1 generation of L3i worms expressing the positive selection marker (GFP) were sorted manually, subjected to single worm genotyping (B) and assayed for feeding behavior (C). Disruption of the $S s-u n c-22$ gene was used as a control. *, p $<0.03$ by Fisher's exact test $(\mathrm{n}=40-200$ from three independent experiments).

(D) $\Delta 7$-DA synthesis is abolished in $S s$-cyp22a9 knockout parasites. Ss-cyp22a9 or Ss-unc-22 (as generation L3i worms were assayed for $\Delta 7$-DA levels as in Figure 3 after treatment with $0.5 \mathrm{mM}$ 
bioRxiv preprint doi: https://doi org/10.1101/2021.09.07.459359; this version posted September 8, 2021. The copyright holder for this preprint (which was not certified by peer review) is the author/funder, who has granted bioRxiv a license to display the preprint in perpetuity. It is made available under aCC-BY 4.0 International license.

507 8-Br-cGMP. $\mathrm{n}=3 \pm$ s.d.; *, $\mathrm{p}<0.03$ by t-test compared to wild type (WT) or Ss-unc-22 worms 508 treated with 8-Br-cGMP.

509 See also Figure 5-figure supplement 1.

510 
A

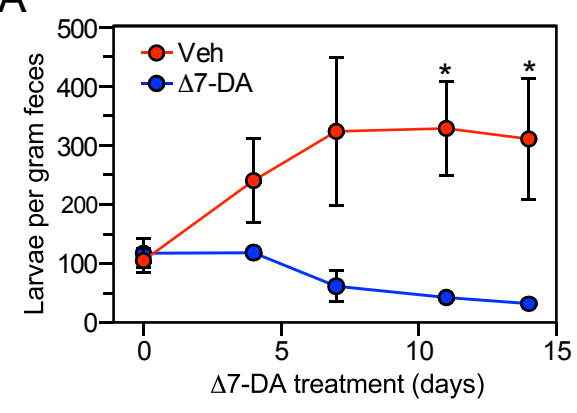

B

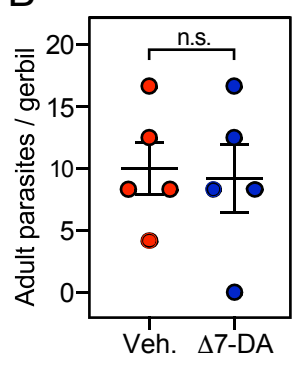

511 Figure 6. $\Delta 7-D A$ suppresses output of fecal larvae in latent, uncomplicated strongyloidiasis.

512 (A) $\Delta 7$-DA treatment reduces fecal larvae by $>90 \%$ in gerbils infected with S. stercoralis.

513 (B) Adult parasite burden in infected gerbils measured at 14 days post-treatment.

$514 \mathrm{n}=5 \pm$ s.e.; ${ }^{*}, \mathrm{q}<0.03$ by Mann-Whitney $\mathrm{U}$ test compared to vehicle. n.s., not significant. 
A

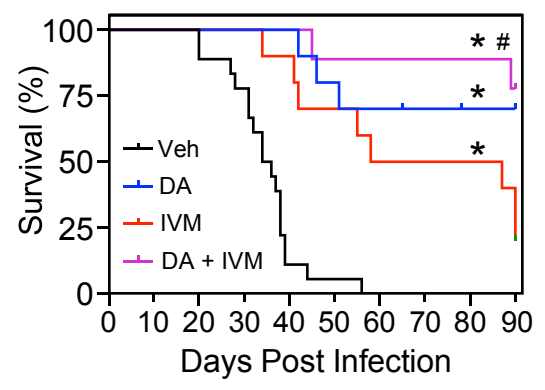

C

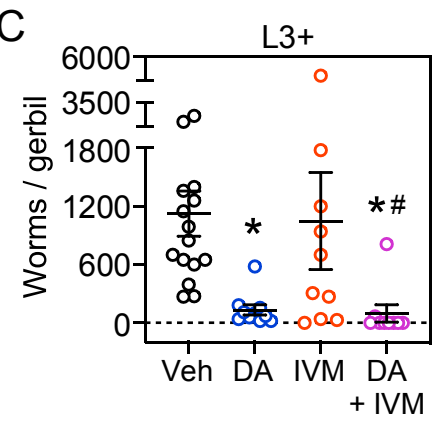

B

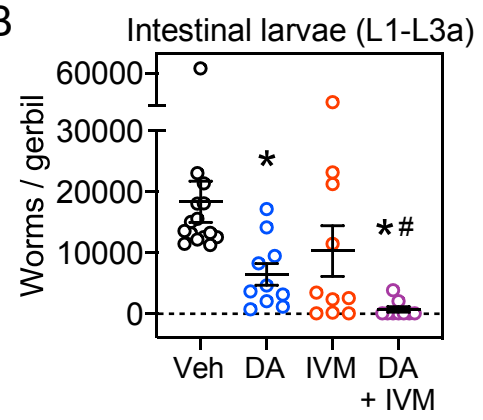

516

517

518

519

520

521

522

523

524

525

526

527

528
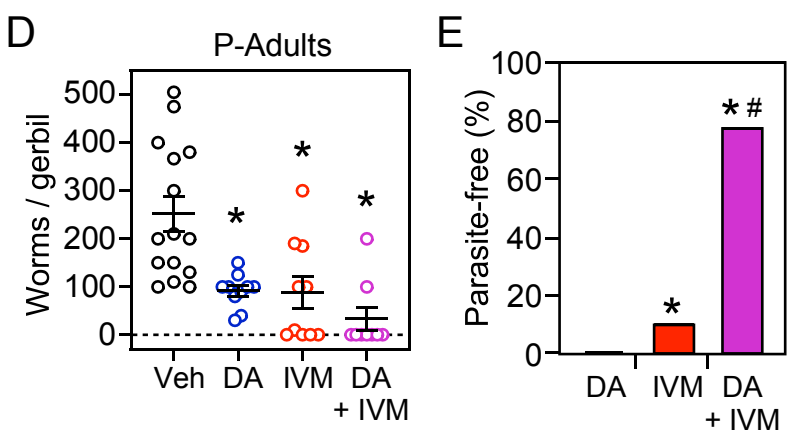

Figure 7. $\triangle 7$-DA and ivermectin act cooperatively to treat disseminated strongyloidiasis

\section{hyperinfection.}

(A) Kaplan-Meier survival curves of hyperinfected gerbils treated with vehicle (Veh), $\Delta 7-\mathrm{DA}$, and/or ivermectin (IVM). Sample sizes: $\mathrm{n}=18$ (Veh), 10 (DA), 10 (IVM), 9 (DA+IVM); q< 0.03, by log-ranked test compared to treatment with vehicle $\left(^{*}\right)$ or ivermectin alone (\#).

(B-D) Parasite burden at various lifecycle stages from the hyperinfected gerbil treatment groups shown in (A). At the time of death, live intestinal L1-L3a (B), L3+ (C), and adult (D) parasites were counted. Sample sizes: $\mathrm{n}=15$ (Veh), 10 (DA), 10 (IVM), 9 (DA+IVM); $\mathrm{q}<0.03$, by MannWhitney $U$ test compared to treatment with vehicle $\left(^{*}\right)$ or ivermectin alone (\#).

(E) Co-treatment with $\triangle 7-\mathrm{DA}$ and ivermectin eradicates parasites in hyperinfected gerbils. Animals from (A) with no detectable parasites in any part of the body after 70 days of treatment were scored as parasite-free. Notably, all the animals in the co-treatment group that survived hyperinfection (shown in (A)) were found to be parasite-free. Sample sizes: n=10 (DA), 10 (IVM), 
$5299(\mathrm{DA}+\mathrm{IVM}) ; \mathrm{q}<0.03$, by Fisher's exact test compared to treatment with vehicle $(*)$ or ivermectin

530 alone (\#). DA, $50 \mu \mathrm{M} \Delta 7$-DA administered in drinking water; IVM, $300 \mu \mathrm{g} / \mathrm{kg}$ ivermectin by i.p.

531 injection.

532 See also Figure 7-figure supplement 1.

533 


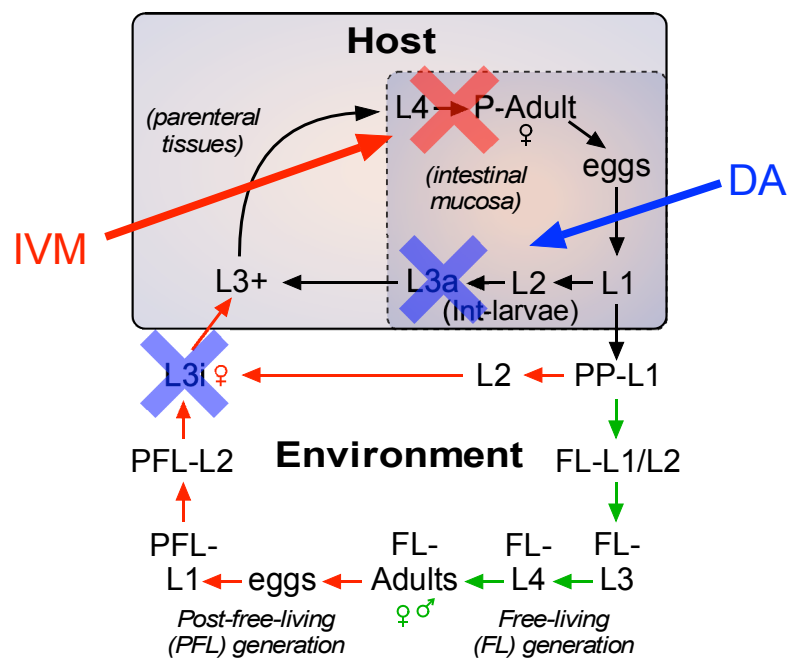

534 Figure 8. Strategy for using DAF-12-based therapeutics to treat strongyloidiasis.

535 Administration of DAF-12 ligands like $\Delta 7$-DA disrupt the lifecycle of nematode parasites by

536 preventing the development of the infective L3i and L3a worms, where DAF-12 is normally

537 unliganded. Pharmacologic activation of DAF-12 with $\Delta 7-\mathrm{DA}$ prevents both environmental

538 infection and autoinfection, which are essential features of the latent and hyperinfection forms of

539 strongyloidiasis. In contrast, ivermectin kills only the actively developing stages and therefore is

540 unable to target developmentally-quiescent infective L3i and L3a larvae. For this reason, the

541 combination of the DAF-12 ligand and ivermectin achieves a synergistic, double blockade of the

542 lifecycle. 

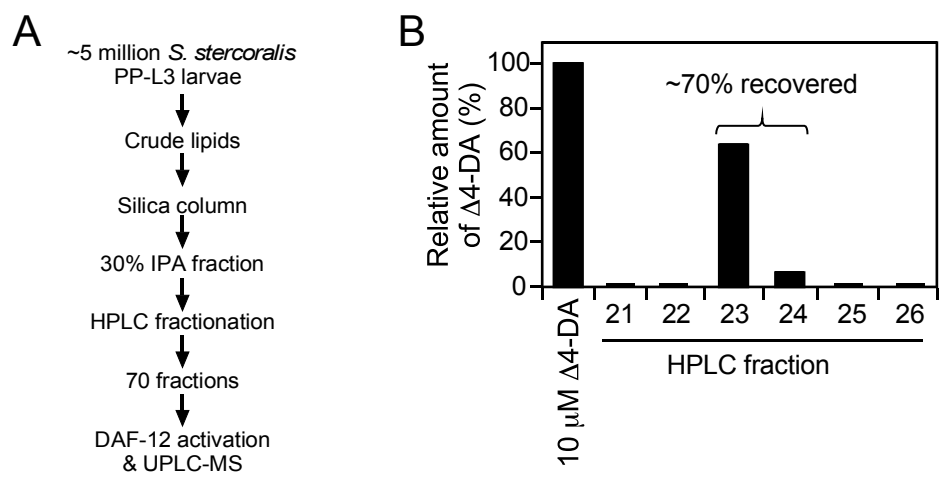

Figure 2-figure supplement 1. Strategy for activity-based, DAF-12 ligand purification in $S$.

546

547

548

549

550

551

552

553

554

\section{stercoralis.}

(A) DAF-12 ligand purification scheme. (B) Determination of purification efficacy. To estimate the efficiency of DA purification, $10 \mathrm{mM}$ of $\Delta 4$-DA was added as a standard to $\sim 2$ million $C$. elegans daf9daf12 worms, a mutant strain that lacks endogenous DA. Following extraction, the lipid fraction was further purified by HPLC. The amount of $\triangle 4$-DA in each of the 70 collected HPLC fractions was analyzed by LC/MS in negative SIM mode at m/z 413. Shown are the only fractions containing detectable $\Delta 4$-DA. Up to $70 \%$ of the $\Delta 4$-DA was recovered, the majority of which was in Fraction 23. The experiment was repeated twice. 


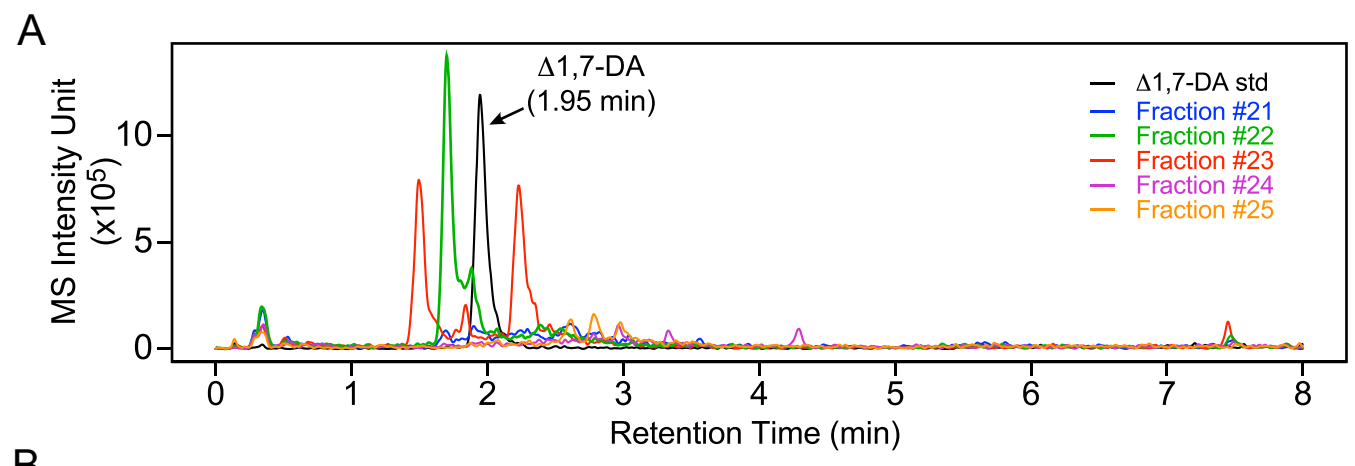

B

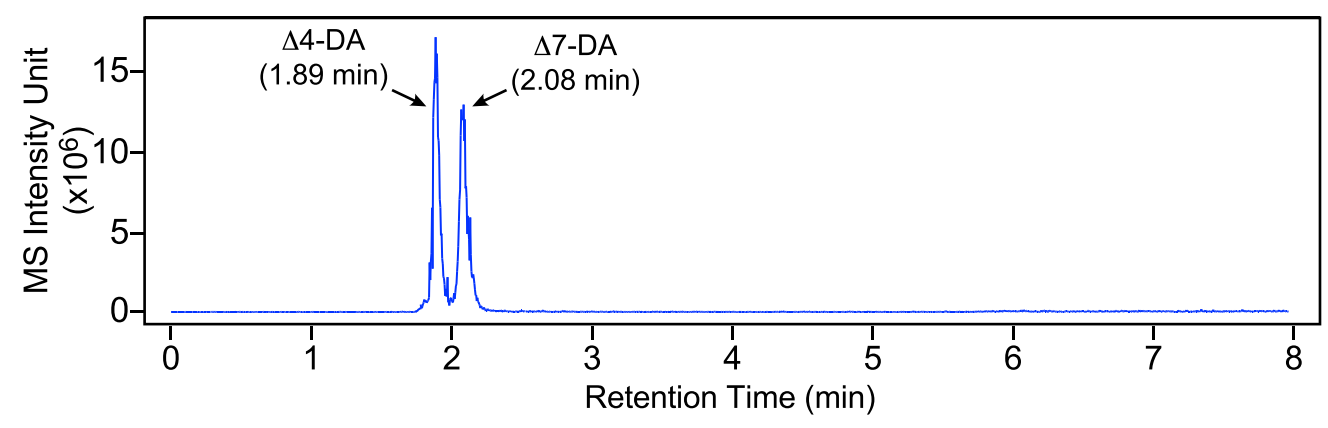

557 analyzed by UPLC-MS in negative SIM mode at m/z 411 and compared to the $\Delta 1,7-\mathrm{DA}$ standard.

560 is undetectable L3 parasites. $\Delta 4$-DA and $\Delta 7$-DA standards were separated and detected by UPLC-

$561 \mathrm{MS}$ in negative SIM mode at $\mathrm{m} / \mathrm{z} 413$. Although $\Delta 4-\mathrm{DA}$ has the same molecular mass as $\Delta 7-\mathrm{DA}$, 562 the two compounds can be completely separated by retention times. Notably, the retention time of 563 the peak in the active fraction shown in Figure 2 only matches $\Delta 7-\mathrm{DA}$. 


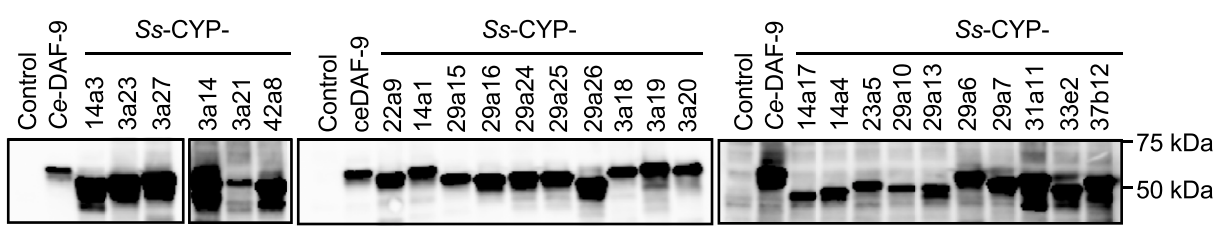

564 Figure 4-figure supplement 1. Expression of the 26 S. stercoralis cytochrome P450 enzymes

565 in insect $\mathbf{S f 9}$ cells.

566 P450 enzymes were fused to C-terminal HA tags and detected by immunoblot using an anti-HA

567 antibody. The $C$. elegans DAF-9 enzyme is shown as a positive control. The experiment was

568 repeated 3 times. 

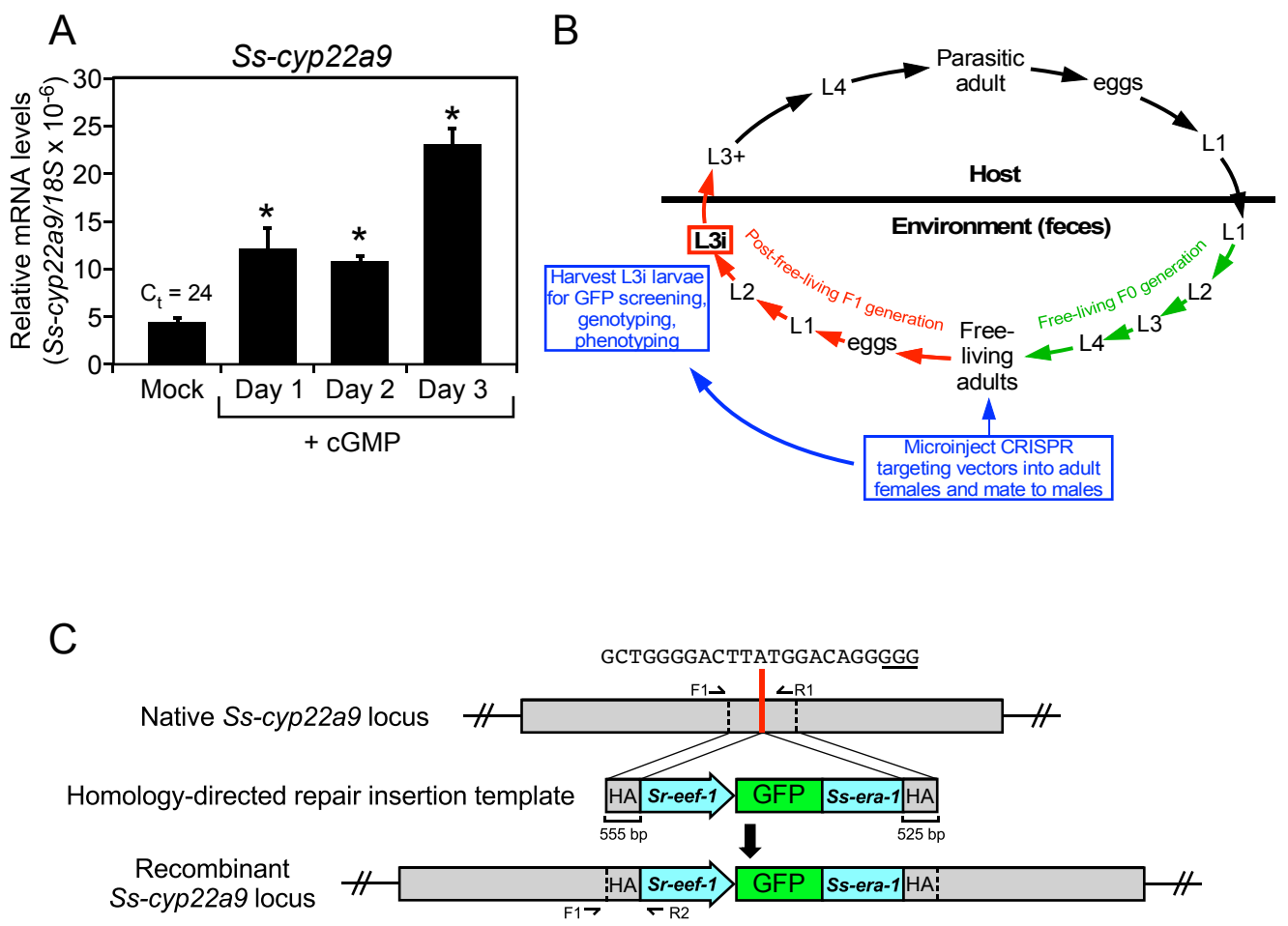

Figure 5-figure supplement 1. In vivo characterization of the DAF-9 homolog (Ss-CYP22a9)

\section{in S. stercoralis.}
(A) Ss-cyp22a9 mRNA expression is increased by cGMP in L3i larvae. L3i larvae were treated as in Figure 5 and $S s$-cyp22a9 mRNA levels were measured by qPCR and compared to $18 \mathrm{~S}$ rRNA levels. Results expressed as means \pm S.D. from technical triplicates. ${ }^{*}, \mathrm{p}<0.01$ by t-test. Duplicate experiments were performed with similar results.

(B) CRISPR knockout strategy for targeting Ss-cyp22a9 in S. stercoralis: Free-living adult females were microinjected with CRISPR targeting vectors (for either NHEJ or HDR gene editing) and allowed to mate. F1 progeny were grown to the L3i stage and GFP-positive worms were phenotyped and genotyped.

(C) Homology-directed repair targeting vector for Ss-cyp22a9 CRISPR. Red line indicates position of the insertion repair template in the P450 domain of the Ss-cyp22a9 gene (SSTP_0001032100).

The guide sequence and PAM site (underlined) are shown. The recombinant locus expresses a 
582 GFP marker from the $S r$-eef-1 promoter fused to the 3'-UTR of the $S s$-era-1 gene. F, R, forward

583 and reverse primers, respectively; HA, homology arms. WT and recombinant alleles produce 1052

584 bp and 823 bp products with F1/R1 and F1/R2, respectively. 


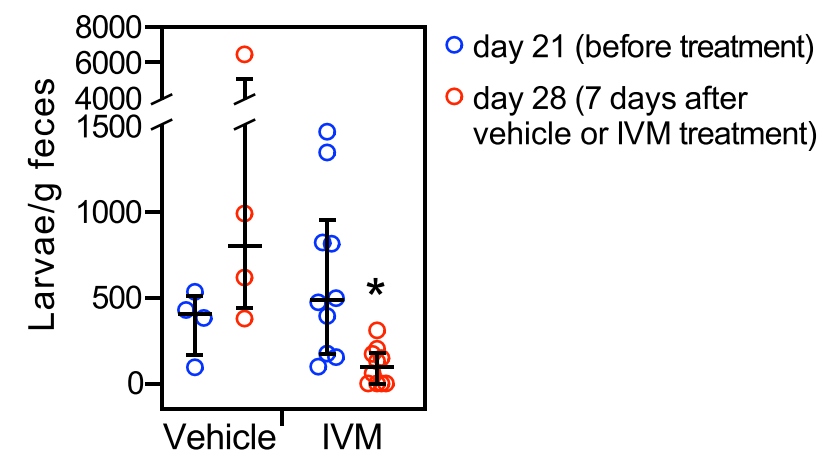

585 Figure 7-figure supplement 1. Ivermectin mimics the treatment of a human S. stercoralis 586 hyperinfection in gerbil models.

587 Vehicle or ivermectin (300 mg/kg) was injected i.p. into S. stercoralis hyperinfected gerbils at 21

588 days post-infection and fecal larval output was measured 7 days later. Ivermectin (IVM) treatment 589 results in $\sim 40 \%$ decrease in fecal larval output, but does not permanently eliminate parasites. $\mathrm{n}=$ 5904 for vehicle, 10 for IVM treatment groups. *, $\mathrm{q}<0.03$ comparing to the day 21 data, by Mann591 Whitney Wilcox test corrected with fdr method. Error bars represent the median $+/-$ interquartile. 
594 Table 1. Detection methods for the compounds in this study.

\begin{tabular}{|c|c|c|c|c|}
\hline Steroids & $\begin{array}{l}\text { Retention time } \\
(\mathrm{min})\end{array}$ & $\begin{array}{l}\text { MS detection } \\
\text { mode }\end{array}$ & $\begin{array}{l}\text { Parent ion } \\
(\mathrm{m} / \mathrm{z})\end{array}$ & $\begin{array}{l}\text { Product ion } \\
(\mathrm{m} / \mathrm{z})\end{array}$ \\
\hline$\Delta 7-\mathrm{DA}$ & 2.1 & Negative SIM & 413 & $\mathrm{~N} / \mathrm{A}$ \\
\hline$\Delta 4-\mathrm{DA}$ & 1.9 & Negative SIM & 413 & $\mathrm{~N} / \mathrm{A}$ \\
\hline$\Delta 1,7-\mathrm{DA}$ & 2.0 & Negative SIM & 411 & N/A \\
\hline$\triangle 7-\mathrm{DA}-\mathrm{PA}$ & 2.1 & Positive MRM & 505 & 487 \\
\hline$\left[{ }^{13} \mathrm{C}\right]-\Delta 7-\mathrm{DA}-\mathrm{PA}$ & 2.1 & Positive MRM & 508 & 490 \\
\hline$\left[{ }^{2} \mathrm{H}\right]$-7-dehydrocholesterol & 3.9 & Positive MRM & 374 & 109 \\
\hline$\left[{ }^{2} \mathrm{H}\right]$-lathosterone & 4.1 & Positive MRM & 392 & 109 \\
\hline
\end{tabular}

Table 2. Sample preparation for $\Delta 7-D A$ quantification in S. stercoralis.

\begin{tabular}{|c|c|c|c|}
\hline Parasite sample & Amount & Lysis method & Standard preparation \\
\hline FL-L1/L2 & 200,000 & Sonication & $\begin{array}{l}100 \mathrm{nM} \Delta 7-\mathrm{DA} \text { compound spiked in } \\
200,000 \mathrm{FL}-\mathrm{L} 1 / \mathrm{L} 2\end{array}$ \\
\hline FL-L3 & 50,000 & Sonication & $\begin{array}{l}100 \mathrm{nM} \Delta 7-\mathrm{DA} \text { compound spiked in } \\
50,000 \mathrm{FL}-\mathrm{L} 3\end{array}$ \\
\hline FL-adult & 5,000 & Sonication & $\begin{array}{l}100 \mathrm{nM} \Delta 7-\mathrm{DA} \text { compound spiked in } \\
5,000 \mathrm{FL} \text {-adults }\end{array}$ \\
\hline PFL-L1 & 200,000 & Sonication & $\begin{array}{l}100 \mathrm{nM} \Delta 7-\mathrm{DA} \text { compound spiked in } \\
200,000 \text { PFL-L1 }\end{array}$ \\
\hline L3i & 50,000 & Sonication & $\begin{array}{l}100 \mathrm{nM} \Delta 7-\mathrm{DA} \text { compound spiked in } \\
50,000 \mathrm{~L} 3 \mathrm{i}\end{array}$ \\
\hline $\mathrm{L} 3+$ & 1,000 & Proteinase $\mathrm{K}$ & $\begin{array}{l}100 \mathrm{nM} \Delta 7-\mathrm{DA} \text { compound spiked in } \\
1,000 \mathrm{~L} 3+\end{array}$ \\
\hline P-adult & 500 & Proteinase K & $\begin{array}{l}100 \mathrm{nM} \Delta 7-\mathrm{DA} \text { compound spiked in } \\
500 \mathrm{FL} \text {-adults }\end{array}$ \\
\hline Intestinal L1-L3a & 5,000 & Proteinase $\mathrm{K}$ & $\begin{array}{l}100 \mathrm{nM} \Delta 7-\mathrm{DA} \text { compound spiked in } \\
5,000 \text { Int-larvae }\end{array}$ \\
\hline cGMP treated L3i & 1,000 & Proteinase K & $\begin{array}{l}100 \mathrm{nM} \Delta 7-\mathrm{DA} \text { compound spiked in } \\
1,000 \mathrm{~L} 3 \mathrm{i}\end{array}$ \\
\hline
\end{tabular}


600 Table 3. Oligo sequences used in this study.

\begin{tabular}{|c|c|c|}
\hline & Sequence & Description \\
\hline forward & GGCATCACCATACAAAACAG & $\begin{array}{l}\text { Ss-cyp } 22 a 9 \text { wild type } \\
\text { allele genotyping }\end{array}$ \\
\hline reverse & TTTGTATGAGGAGGGTTGTG & \\
\hline forward & GGCATCACCATACAAAACAG & $\begin{array}{l}\text { Ss-cyp } 22 a 9 \mathrm{KO} \text { allele } \\
\text { genotyping }\end{array}$ \\
\hline reverse & CATCACATTCATCAAAAGTCCACT & \\
\hline forward & TCCTGGCCAGTGCTAATGTTATT & Ss-cyp $22 a 9$ qPCR \\
\hline reverse & CTATTTGGACGGGATGAGAAGACT & \\
\hline forward & TGGTGCATGGCCGTTCTTA & Ss-18SRNA qPCR \\
\hline reverse & CTCGCTCGTTATCGGAATCAA & \\
\hline forward & GCTGGGGACTTATGGACAGGgttttagagctagaaatagcaag & $\begin{array}{l}\text { sgRNA expression } \\
\text { plasmid }\end{array}$ \\
\hline reverse & /5phos/CATTGTATTGGATGGCAATC & targeting Ss-cyp22a9 \\
\hline
\end{tabular}




\section{MATERIALS AND METHODS}

603

604

605

606

607

608

609

610

611

612

613

614

615

616

617

618

619

620

621

622

623

624

\section{Animal husbandry}

All animal experiments were approved by the University of Texas Southwestern Medical Center Institutional Animal Care and Use Committee (IACUC) and are listed under animal protocol numbers 2016-101500 and 2018-102369. All protocols, as well as routine husbandry care of the animals, were conducted in strict accordance with the Guide for the Care and Use of Laboratory Animals of the National Institutes of Health (NationalResearchCouncil, 2011). Male dogs of 6 18 months were purchased from Oak Hill Genetics (Ewing, IL) and infected with 3000 L3i of $S$. stercoralis and were orally administrated $0.25 \sim 1.2 \mathrm{mg} / \mathrm{kg}$ prednisolone daily to maintain parasite production in feces. Male Mongolian gerbils (TUM/MON strain) of $6 \sim 9$ weeks were purchased from Charles Rivers (Wilmington, MA) and used as described below.

\section{Reagents}

Dafachronic acids were synthesized as reported (Basu et al., 2015; Mahanti et al., 2014; Sharma et al., 2009). Methylprednisolone acetate (MPA) injection suspension (Zoetis, Parsippany, NJ), ketoconazole (Sigma, St. Louis, MO), 8-Br-cGMP (Tocris, Minneapolis, MN), ivermectin (Merial Limited, Duluth, GA) were purchased as cited. Solvents for lipid extraction and liquid chromatography (toluene, hexanes, isopropanol, methanol, water, and acetonitrile) were purchased from Fisher Scientific (Waltham, MA). Eluent additives for LC-MS (formic acid or NH4Ac) were purchased from Sigma (St. Louis, MO).

\section{S. stercoralis culture}

The wild type (UPD strain) of S. stercoralis was maintained in purpose-bred dogs (Oak Hill Genetics, Ewing, IL) and free-living stages of S. stercoralis were prepared from coproculture of the dog feces as described (Lok, 2007). Briefly, post-parasitic worms at the noted stages were 
625

626

627

628

629

630

631

632

633

634

635

636

637

638

639

640

641

642

643

644

645

646

647

obtained using the Baermann technique (Lok, 2007) from daily collected dog feces (for PP-L1/L2); $24 \mathrm{hr}$ coproculture at $18^{\circ} \mathrm{C}$ (for FL-L3); $72 \mathrm{hr}$ coproculture at $21^{\circ} \mathrm{C}$ (for FL-adults and PFL-L1); and 7-day coproculture at $25^{\circ} \mathrm{C}$ (for L3i). The parasitic stages of $S$. stercoralis were acquired from Mongolian gerbils (Charles Rivers, Wilmington, MA) as described (Lok, 2007; Stoltzfus et al., 2012). To obtain L3+, gerbils infected with 10,000 L3i larvae were sacrificed 3 days post infection. After removing the intestinal tract, parenteral tissues were minced and L3+ were collected by the Baermann technique at $37^{\circ} \mathrm{C}$. To collect P-adults, intestinal L1-L3, and L3a, gerbils were hyperinfected by an initial inoculation of 4,000 L3i and administration of $2 \mathrm{mg}$ MPA, followed by weekly injections of $2 \mathrm{mg}$ MPA for 4 weeks. After sacrificing the gerbils, P-females and intestinal larval stages were collected by hanging the small and large intestines in cylinders filled with saline water supplemented with $0.5 \mathrm{mg} / \mathrm{ml}$ gentamicin (Sigma, St. Louis, MO) at $37^{\circ} \mathrm{C}$, which allows the parasites to migrate out of the tissues and settle down at bottom of the cylinders. The parasites were then collected and purified by manually removing each stage under a dissection scope. Collected parasites were then washed 5 times with M9 buffer containing an antibiotic cocktail containing 100 units penicillin, $100 \mathrm{ug} / \mathrm{ml}$ streptomycin (ThermoFisher, Waltham, MA), and 50 $\mu \mathrm{g} / \mathrm{ml}$ gentamicin (Sigma, St. Louis, MO) and stored in $-80^{\circ} \mathrm{C}$ for lipid extraction.

\section{Lipid fractionation and ligand identification}

Endogenous DAF-12 ligands were purified from the lipid fractions of PP-L3 as outlined (Figure 2-figure supplement 1A). Briefly, $\sim 5$ million PP-L3s were obtained from $\sim 100 \mathrm{~kg}$ dog feces as described above. The worms were pooled and sonicated in $0.9 \% \mathrm{NaCl}$, from which crude lipids were extracted by the Folch method (Folch et al., 1957). The crude lipids were dissolved in toluene and $0.1 \%$ acetic acid and passed through a Sep-Pak Silica SPE columns (Waters, Milford, MA), followed by sequential elution with hexanes, 30\% isopropanol in hexanes, and finally 
648

649

650

651

652

653

654

655

656

657

658

659

660

661

662

663

664

665

666

667

668

669

670

methanol, all of which contains $0.1 \%$ acetic acids. The lipids in the $30 \%$ isopropanol fraction were then fractionated with semi-preparative high-performance liquid chromatography (HPLC) with a C18 column (Luna $5 \mu \mathrm{m} \mathrm{C18} 100 \AA \AA, 250$ x $10 \mathrm{~mm}$, Phenomenex, Torrance, CA) at a flow rate of $3.5 \mathrm{ml} / \mathrm{min}$. The mobile phases were water (A) and acetonitrile (B), both containing $0.1 \%$ formic acid. The following gradient was run for a total of $70 \mathrm{~min}$ : $0-15 \mathrm{~min}$ with $50 \sim 100 \%$ (B); $15 \sim 70$ min with $100 \%$ (B). Eluted fractions were collected every minute and dried under nitrogen gas and dissolved in ethanol.

HPLC fractions containing endogenous Ss-DAF-12 ligands were first identified using a cellbased reporter assay (Wang et al., 2009). Briefly, COS-7 cells (ATCC, Manassas, VA) cultured in DMEM (Thermofisher, Waltham, MA) supplemented with 10\% FBS (Gemini Bio, West Sacramento, CA) were co-transfected with plasmids expressing the S. stercoralis DAF-12 ligand binding domain fused in frame with the DNA binding domain from C. elegans DAF-12 (pCMXCeSs-DAF-12, to increase expression efficiency) (Wang et al., 2009), the lit-1 kinase DAF-12 reporter plasmid (pNL3.1-DAF-12RE-lit1, fused to Nanoluc luciferase) and the control reporter plasmid (pGL4.53, pgk promoter fused to firefly luciferase). The cells were split into 384 well plates and treated with $10 \mathrm{nM} \triangle 7$-DA or the HPLC fractions. After $24 \mathrm{hr}$, Nanoluc and firefly luciferase activities were measured by Nano-Glo Dual-luciferase kits (Promega, Madison, WI) on a Victor V plate reader (Perkin Elmer, Waltham, MA). Relative luciferase units (RLU) were then calculated by normalizing the Nanoluc luciferase activity to the firefly luciferase activity.

To characterize the chemical identity of the Ss-DAF-12 ligand, isolated HPLC fractions were subjected to ultra-performance liquid chromatography tandem with mass spectrometry (UPLCMS) using a Shimadzu LC instrument (Shimadzu Scientific Instruments, Inc., Columbia, MD) in tandem with a Sciex 6500 Triple Quad mass spectrometer (AB Sciex LLC, Framingham, MA). 
671 The HPLC fractions were diluted in methanol containing $100 \mathrm{nM}$ chenodeoxycholic acid- ${ }^{2} \mathrm{H}_{4}(\mathrm{~d} 4-$

672 CDCA, Sigma, St. Louis, MO) as an internal control of retention times between different runs.

673 The lipids were then loaded onto a C18 column (Kinetex $1.3 \mu \mathrm{m} \mathrm{C18} 100 \AA$, LC Column 50 x

following gradient was run for a total of $8 \mathrm{~min}$ : $60 \sim 80 \%$ of (B) over $0 \sim 2 \mathrm{~min} ; 80 \%$ (B) over $2 \sim 4$ with the lipid fractions.

\section{Quantitative analysis of $\Delta 7$-DA levels in $S$. stercoralis}


694

695

696

697

698

699

700

701

702

703

704

705

706

707

708

709

710

711

712

713

714

715

716

triphenylphosphine, $3.3 \mathrm{mM}$ 2,2'-dipyridyl disulfide, and $333 \mathrm{ng} / \mathrm{ul}$ 2-picolylamine at $60^{\circ} \mathrm{C}$ for 20 min (Li et al., 2013). The $\triangle 7$-DA-PA was then analyzed by UPLC-MS. The UPLC method was same as described above and $\Delta 7$-DA-PA compounds were detected in positive multiple reaction monitoring $(\mathrm{MRM})$ mode with $\mathrm{m} / \mathrm{z}$ transition $505 \rightarrow 487$. DA quantification was then performed with the software MultiQuant (AB Sciex LLC, Framingham, MA) by comparing $\Delta 7$-DA-PA peak areas in the parasite samples with those of $100 \mathrm{nM} \Delta 7$-DA standards.

\section{Biosynthesis of $\Delta 7$-DA from dietary cholesterol in S. stercoralis}

PP-L1 stages of S. stercoralis were prepared from dog feces as described above and cultured on NGM plates modified for metabolic tracing of isotope-labeled cholesterol supplemented in the diet. Briefly, HB101 bacteria were cultured overnight in cholesterol-free medium $(10 \mathrm{~g} / \mathrm{L}$ etherextracted peptone in DMEM) and prepared as $10 \times$ concentrates supplemented with $140 \mu \mathrm{M}$ naturally labeled (i.e., ${ }^{12} \mathrm{C}$ ) or ${ }^{13} \mathrm{C}_{3}$ (Cambridge Isotope Laboratories, Tewksbury, MA) labeled cholesterol. The bacteria concentrates were then spotted on cholesterol-free NGM plates $(3 \%$ agarose in S-basal) to form bacterial lawns. Approximately 10,000 PP-L1 larvae were cultured on the plates at $18^{\circ} \mathrm{C}$ for $24 \mathrm{~h}$ and collected and analyzed as described above for $\Delta 7-\mathrm{DA}-\mathrm{PA}-{ }^{13} \mathrm{C}_{3}$ levels by UPLC-MS in the positive MRM mode with $\mathrm{m} / \mathrm{z}$ transition $508 \rightarrow 490$.

\section{In vitro enzyme activity assays}

Candidate enzyme genes (Stoltzfus et al., 2012) were cloned and each gene expressed in Sf9 cells (ATCC, Manassas, VA), which are cultured with Sf 900 III medium (Thermofisher, Waltham, MA). A human CYP450 oxidoreductase (hOR) was co-expressed to facilitate electron transport required for the enzyme activities (Motola et al., 2006). Enzyme coding sequences were tethered with a C-terminal HA tag in pFastBac Dual baculoviral vectors (Invitrogen, Waltham, MA) using $\mathrm{Ph}$ or p10 expression cassettes for hOR or other enzymes, respectively. Baculovirus was prepared 
717

718

719

720

721

722

723

724

725

726

727

728

729

730

731

732

733

734

735

736

737

738

739

according to manufacturer's instructions followed by infection of Sf9 cells. For DAF-36 and DHS16 homologs, the microsomes expressing the enzymes were purified from the infected Sf9 cells with a Microsome Isolation Kit (Abcam, Cambridge, MA). Microsomes were incubated with an NADPH regeneration system (Promega, Madison, WI) in presence or absence of $100 \mu \mathrm{M}$ substrate compounds at $37^{\circ} \mathrm{C}$ for $16 \mathrm{hrs}$. For CYP activities, infected Sf9 cells were incubated with $10 \mu \mathrm{M}$ substrate compound at $28^{\circ} \mathrm{C}$ for $24 \mathrm{hrs}$. The substrate compounds for DAF-36, DHS-16 and CYPs were cholesterol- ${ }^{2} \mathrm{H}_{7}$, lathosterol- ${ }^{2} \mathrm{H}_{7}$ (Avanti Polar Lipids, Inc., Alabaster, AL), and lathosterone (Steraloids, Inc., Newport, RI), respectively. Lipids were then extracted from microsomes (for DAF-36s and DHS-16s) or Sf9 cells (for CYPs) by the Folch method and loaded onto a C18 column (Kinetex $1.3 \mu \mathrm{m} \mathrm{C18} 100 \AA \AA$, LC Column 50 x 2.1mm, Phenomenex, Torrance, CA) at a $0.4 \mathrm{ml} / \mathrm{min}$ flow rate. The mobile phase consisted of (A) water:acetonitrile $(9: 1)$ and (B) acetonitrile:water (99:1), both containing 2 mM NH4Ac. For measuring the activities of the DAF36 and DHS-16 homologs, the following gradient was run for a total of 8 min: $80 \sim 99 \%$ (B) for 0 2 $\mathrm{min}$; 99\% (B) for 2 8 $\mathrm{min}$. Enzyme products were detected in positive MRM mode with $\mathrm{m} / \mathrm{z}$ transition $374 \rightarrow 109$ (7-dehydrocholesterol- ${ }^{2} \mathrm{H}_{7}$ ) and $392 \rightarrow 109$ (lathosterone- ${ }^{2} \mathrm{H}_{7}$ ). For CYP activities, $\triangle 7$-DA was analyzed by the UPLC-MS methods described above.

\section{L3i activation assay}

Reactivation of L3i developmental arrest was assayed by monitoring feeding activity as described (Albarqi et al., 2016; Cheong et al., 2021). Briefly, L3i larvae prepared as described above were suspended in M9 buffer at 100 larvae/96 well. The larvae were treated with $0.5 \mathrm{mM}$ 8-Br-cGMP (Torcis, Minneapolis, MN), $1 \mu \mathrm{M} \Delta 7$-DA and/or $25 \mu \mathrm{M}$ of ketoconazole (Sigma, St. Louis, MO) at $37^{\circ} \mathrm{C}$ for $22 \mathrm{hrs}$. To visualize the feeding behaviors, worms were incubated with $200 \mu \mathrm{g} / \mathrm{ml}$ of Alexa 594 fluorescent dye (Thermofisher, Waltham, MA) at $37^{\circ} \mathrm{C}$ for another 3 hours, 
washed and observed under fluorescent microscope. Worms with internal red fluorescence were scored as feeding larvae. For $\Delta 7$-DA levels, worms were suspended in M9 buffer at 4000 larvae/ml and treated for the indicated times. $\triangle 7$-DA levels were then analyzed by UPLC-MS as described above.

\section{QPCR}

QPCR was performed by SYBR green method as reported (Wang et al., 2015). Briefly, 10,000-20,000 L3i larvae were pelleted and RNA extracted with RNA-STAT60 reagent (Amsbio, Cambridge, MA). Total RNA was digested with Turbo-DNAase (Ambion Inc., Austin, TX) and purified with RNeasy Mini Kits (Qiagen, Germantown, MD) to remove genomic DNA. Following reverse transcription, $S s-c y p 22 a 9$ and $S s-18 S$ rRNA levels were analyzed by QPCR (primer sequences in Table S3) and presented as relative mRNA levels (Ss-cyp22a9/Ss-18S rRNA).

\section{Immunoblotting}

Western immunoblotting was performed following standard protocol. Sf9 cells expressing SsCYP450 enzymes were pelleted and lysed in Laemmli sample buffer by sonication followed by 5 min in a boiling water bath and 2 min on ice. The samples were then analyzed by $10 \%$ SDS-PAGE transferred to nitrocellulose membrane, and immunoblotted with the mouse anti-HA antibody [HA.C5] (Abcam, Cambridge, MA) followed by HRP-conjugated anti-mouse antibody (Abcam, Cambridge, MA). The membranes were then imaged by ImageQuant LAS4000 luminescent Image Analyzer (GE Healthcare, Chicago, IL) following an incubation with ECL chemiluminescent substrate reagent kit (ThermoFisher, Waltham, MA).

\section{CRISPR gene disruption}

To knock out expression of the gene encoding the DAF-9 ortholog, Ss-cyp22a9, CRISPR methods developed specifically for S. stercoralis were employed as outlined (Figure 5-figure 
763

764

765

766

767

768

769

770

771

772

773

774

775

776

777

778

779

780

781

782

supplement 1B, C) and previously reported (Cheong et al., 2021; Gang et al., 2017; Lok, 2019). Briefly, an sgRNA targeting Ss-cyp22a9 was designed by GPP sgRNA Designer (Broad Institute) and CHOPCHOP (http://chopchop.cbu.uib.no/). Top candidates with 5'-(N) ${ }_{18} \mathrm{GG}-3$ ' formats from both designs were compared to the $S$. stercoralis genome (https://parasite.wormbase.org/Strongyloides_stercoralis_prjeb528/Info/Index/) for off-targeting analysis. The sequence 5'-GCTGGGGACTTATGGACAGG-3', was selected as only targeting the Ss-cyp22a9 locus within the entire $S$. stercoralis genome and cloned into the gRNA vector pML60 (Gang et al., 2017). Ss-unc-22 sgRNA (gift from Dr. Ellisa A. Hallem, UCLA) was used as a control. CRISPR gene editing was accomplished using both homologous directed repair (HDR) and non-homologous end joining (NHEJ) and vectors as previously published (Gang et al., 2017; Lok, 2019). For HDR, a homology repair template was made by sewing 500 bp homology arms flanking the Cas9 cutting site at the Ss-cyp22a9 locus with a GFP expressing cassette (Sr-eef1p::GFP::era, from pPV529) (Figure 5-figure supplement 1C) by fusion PCR. At least fifty freeliving females of $S$. stercoralis were microinjected with plasmids expressing sgRNA (60 ng/ul), Cas9 (pPV540, $20 \mathrm{ng} / \mathrm{ul}$ ) and the homology repair template $(10 \mathrm{ng} / \mathrm{ul})$. Injected females were then placed with adult males in the fecal culture as described (Cheong et al., 2021; Gang et al., 2017; Lok, 2019). The F1 progenies from these matings were collected after 7 -day fecal culture at $25^{\circ} \mathrm{C}$ with the Baermann technique. The GFP positive L3i larvae from the F1 progenies were manually picked and treated with $0.5 \mathrm{mM} 8-\mathrm{Br}-\mathrm{cGMP}$ or $1 \mu \mathrm{M} \Delta 7-\mathrm{DA}$ in M9 buffer at $37^{\circ} \mathrm{C}$ for $22 \mathrm{hrs}$. The feeding behaviors were then visualized as described above and genotyped individually by single worm PCR with the primers listed in Table 3.

In a second set of experiments, NHEJ CRISPR was used to produce enough Ss-cyp22a9 knock-out worms for detection of endogenous $\Delta 7$-DA. As described above, 50 free-living females 
of S. stercoralis were microinjected with plasmids expressing Cas9 (pPV540, $20 \mathrm{ng} / \mathrm{ul}$ ) and sgRNAs against $S s-c y p 22 a 9$ or $S s-u n c-22(60 \mathrm{ng} / \mathrm{ul})$. Following a fecal culture with free-living males at $25^{\circ} \mathrm{C}$ for 7 days, the resulting L3i larvae were collected by the Baermann technique and treated by $0.5 \mathrm{mM}$ 8-Br-cGMP in M9 buffer for 3 days. Levels of $\Delta 7$-DA were analyzed by UPLCMS as described above.

\section{Gerbil models of $\boldsymbol{S}$. stercoralis infection}

Uncomplicated and hyperinfection cases of strongyloidiasis were established in Mongolian gerbils (Charles Rivers, Wilmington, MA) as described (Nolan et al., 1993). For uncomplicated strongyloidiasis, gerbils were subcutaneously injected with 1000 L3i S. stercoralis larvae. On day 21 post the infection, the gerbils were switched to drinking water containing 5\% sucrose (vehicle) or $50 \mu \mathrm{M} \Delta 7$-DA in $5 \%$ sucrose. We have found that adding a small amount of sucrose does not affect infection, but ensures animals drink appropriate amounts of drug. Fecal larval numbers were monitored bi-weekly for the next 14 days. To establish hyperinfection, gerbils were infected similarly but MPA (2 mg/gerbil) was injected subcutaneously weekly from the time of infection. After 10 days post-infection to allow time for the hyperinfection to occur, $\Delta 7$-DA treatment was begun as noted above. Ivermectin (Merial Limited, Duluth, GA) was intraperitoneally injected at $300 \mathrm{ug} / \mathrm{kg}$ on day 21 post infection. Since $\Delta 7$-DA is rapidly turned over in vivo (Patton et al., 2018), administering it at an earlier time point ensured an adequate amount was delivered. Animals were observed daily for irreversible hyperinfection symptoms that indicate imminent lethality, which include lethargy, hunched back, scruffy hair, lack of activity, and reduced drinking. Animals with such symptoms were sacrificed and autopsy performed to determine end-point parasite numbers as described below. 


\section{Parasite burden analysis}

Parasite numbers in gerbils were determined as described with modifications (Lok, 2007; Nolan et al., 1993; Stoltzfus et al., 2012). For fecal larvae outputs, infected gerbils were housed in wire bottom cages with a layer of dampened paper towel for $8 \mathrm{hrs}$. Feces (15-20 droppings) were collected and macerated in $0.9 \% \mathrm{NaCl}$. The fecal suspension was then filtered through a layer of cheese cloth and an aliquot of the suspension was spotted on NGM plates. After drying, plates were incubated at $37^{\circ} \mathrm{C}$ for $10 \mathrm{~min}$ and living larvae, which leave traces on the plates, were counted and normalized to the feces weight as larvae per gram feces. To determine end-point parasite burden, intestines were cut longitudinally and incubated in $0.9 \% \mathrm{NaCl}$ supplemented with an antibiotic cocktail (100 units $/ \mathrm{ml}$ penicillin, $100 \mu \mathrm{g} / \mathrm{ml}$ streptomycin and $50 \mu \mathrm{g} / \mathrm{ml}$ gentamicin) at $37^{\circ} \mathrm{C}$ for $2-3 \mathrm{hrs}$. The suspension was then filtered through a layer of cheese cloth and an aliquot of the suspension was spotted on NGM plates. Following drying and 10 min incubation at $37^{\circ} \mathrm{C}$, parasitic adults and intestinal L1-L3a larvae were counted. To determine parasite burden of hyperinfected L3+, parenteral tissues were dissected, minced and larvae collected by the Baermann method were counted on watch glasses.

\section{Data analysis}

Data were plotted and analyzed by the indicated statistical tests using GraphPad Prism 8 software to obtain $p$ values. When multiple comparisons were involved, pairwise statistical analyses were first performed to obtain $\mathrm{p}$ values and then adjusted by the false discovery rate method using the p.adjust function in R to obtain q values (https://www.R-project.org/). 


\section{REFERENCES}

830

831

832

833

834

835

836

837

838

839

840

841

842

843

844

845

846

847

848

849

850

851

852

853

854

855

856

857

858

859

860

861

862

863

864

865

866

867

868

869

870

871

872

873

Albarqi, M. M., Stoltzfus, J. D., Pilgrim, A. A., Nolan, T. J., Wang, Z., Kliewer, S. A., Mangelsdorf, D. J., \& Lok, J. B. (2016). Regulation of life cycle checkpoints and developmental activation of infective larvae in Strongyloides stercoralis by dafachronic acid. PLoS Pathog, 12(1), e1005358. https://doi.org/10.1371/journal.ppat.1005358

Antebi, A., Culotti, J. G., \& Hedgecock, E. M. (1998). daf-12 regulates developmental age and the dauer alternative in Caenorhabditis elegans. Development, 125(7), 1191-1205. https://www.ncbi.nlm.nih.gov/pubmed/9477318

Antebi, A., Yeh, W. H., Tait, D., Hedgecock, E. M., \& Riddle, D. L. (2000). daf-12 encodes a nuclear receptor that regulates the dauer diapause and developmental age in C. elegans. Genes Dev, 14(12), 1512-1527. https://www.ncbi.nlm.nih.gov/pubmed/10859169

Ayoade, K. O., Carranza, F. R., Cho, W. H., Wang, Z., Kliewer, S. A., Mangelsdorf, D. J., \& Stoltzfus, J. D. C. (2020). Dafachronic acid and temperature regulate canonical dauer pathways during Nippostrongylus brasiliensis infectious larvae activation. Parasit Vectors, 13(1), 162. https://doi.org/10.1186/s13071-020-04035-z

Basu, D., Mazumder, S., Shi, X., Baydoun, H., Niklas, J., Poluektov, O., Schlegel, H. B., \& Verani, C. N. (2015). Ligand transformations and efficient proton/water reduction with cobalt catalysts based on pentadentate pyridine-rich environments. Angew Chem Int Ed Engl, 54(7), 2105-2110. https://doi.org/10.1002/anie.201409813

Bethke, A., Fielenbach, N., Wang, Z., Mangelsdorf, D. J., \& Antebi, A. (2009). Nuclear hormone receptor regulation of microRNAs controls developmental progression. Science, 324(5923), 95-98. https://doi.org/10.1126/science.1164899

Bisoffi, Z., Buonfrate, D., Montresor, A., Requena-Mendez, A., Munoz, J., Krolewiecki, A. J., Gotuzzo, E., Mena, M. A., Chiodini, P. L., Anselmi, M., Moreira, J., \& Albonico, M. (2013). Strongyloides stercoralis: a plea for action. PLoS Negl Trop Dis, 7(5), e2214. https://doi.org/10.1371/journal.pntd.0002214

Buonfrate, D., Bisanzio, D., Giorli, G., Odermatt, P., Fürst, T., Greenaway, C., French, M., Reithinger, R., Gobbi, F., Montresor, A., \& Bisoffi, Z. (2020). The Global Prevalence of Strongyloides stercoralis Infection. Pathogens, 9(6), 468. https://www.mdpi.com/2076$\underline{0817 / 9 / 6 / 468}$

Buonfrate, D., Requena-Mendez, A., Angheben, A., Munoz, J., Gobbi, F., Van Den Ende, J., \& Bisoffi, Z. (2013). Severe strongyloidiasis: a systematic review of case reports. BMC Infect Dis, 13, 78. https://doi.org/10.1186/1471-2334-13-78

Chen, T. (2008). Nuclear receptor drug discovery. Curr Opin Chem Biol, 12(4), 418-426. https://doi.org/10.1016/j.cbpa.2008.07.001

Cheong, M. C., Wang, Z., Jaleta, T. G., Li, X., Lok, J. B., Kliewer, S. A., \& Mangelsdorf, D. J. (2021). Identification of a nuclear receptor/coactivator developmental signaling pathway in the nematode parasite Strongyloides stercoralis. Proc Natl Acad Sci U S A, 118(8). https://doi.org/10.1073/pnas.2021864118

Folch, J., Lees, M., \& Sloane Stanley, G. H. (1957). A simple method for the isolation and purification of total lipides from animal tissues. J Biol Chem, 226(1), 497-509. https://www.ncbi.nlm.nih.gov/pubmed/13428781

Gang, S. S., Castelletto, M. L., Bryant, A. S., Yang, E., Mancuso, N., Lopez, J. B., Pellegrini, M., \& Hallem, E. A. (2017). Targeted mutagenesis in a human-parasitic nematode. PLoS Pathog, 13(10), e1006675. https://doi.org/10.1371/journal.ppat.1006675 
Gerisch, B., \& Antebi, A. (2004). Hormonal signals produced by DAF-9/cytochrome P450 regulate $C$. elegans dauer diapause in response to environmental cues. Development, 131(8), 1765-1776. https://doi.org/10.1242/dev.01068

Gerisch, B., Rottiers, V., Li, D., Motola, D. L., Cummins, C. L., Lehrach, H., Mangelsdorf, D. J., \& Antebi, A. (2007). A bile acid-like steroid modulates Caenorhabditis elegans lifespan through nuclear receptor signaling. Proc Natl Acad Sci U S A, 104(12), 5014-5019. https://doi.org/10.1073/pnas.0700847104

Hammell, C. M., Karp, X., \& Ambros, V. (2009). A feedback circuit involving let-7-family miRNAs and DAF-12 integrates environmental signals and developmental timing in Caenorhabditis elegans. Proc Natl Acad Sci U S A, 106(44), 18668-18673. https://doi.org/10.1073/pnas.0908131106

Hu, P. J. (2007). Dauer. WormBook, 1-19. https://doi.org/10.1895/wormbook.1.144.1

Jia, K., Albert, P. S., \& Riddle, D. L. (2002). DAF-9, a cytochrome P450 regulating C. elegans larval development and adult longevity. Development, 129(1), 221-231. https://www.ncbi.nlm.nih.gov/pubmed/11782415

Kerlin, R. L., Nolan, T. J., \& Schad, G. A. (1995). Strongyloides stercoralis: histopathology of uncomplicated and hyperinfective strongyloidiasis in the Mongolian gerbil, a rodent model for human strongyloidiasis [corrected]. Int J Parasitol, 25(4), 411-420. https://doi.org/10.1016/0020-7519(94)00154-g

Krolewiecki, A. J., Lammie, P., Jacobson, J., Gabrielli, A. F., Levecke, B., Socias, E., Arias, L. M., Sosa, N., Abraham, D., Cimino, R., Echazu, A., Crudo, F., Vercruysse, J., \& Albonico, M. (2013). A public health response against Strongyloides stercoralis: time to look at soil-transmitted helminthiasis in full. PLoS Negl Trop Dis, 7(5), e2165. https://doi.org/10.1371/journal.pntd.0002165

Li, T. M., Chen, J., Li, X., Ding, X. J., Wu, Y., Zhao, L. F., Chen, S., Lei, X., \& Dong, M. Q. (2013). Absolute quantification of a steroid hormone that regulates development in Caenorhabditis elegans. Anal Chem, 85(19), 9281-9287. https://doi.org/10.1021/ac402025c

Lok, J. B. (2007). Strongyloides stercoralis: a model for translational research on parasitic nematode biology. WormBook, 1-18. https://doi.org/10.1895/wormbook.1.134.1

Lok, J. B. (2019). CRISPR/Cas9 mutagenesis and expression of dominant mutant transgenes as functional genomic approaches in parasitic nematodes. Front Genet, 10, 656. https://doi.org/10.3389/fgene.2019.00656

Long, T., Alberich, M., Andre, F., Menez, C., Prichard, R. K., \& Lespine, A. (2020). The development of the dog heartworm is highly sensitive to sterols which activate the orthologue of the nuclear receptor DAF-12. Sci Rep, 10(1), 11207. https://doi.org/10.1038/s41598-020-67466-9

Ma, G., Wang, T., Korhonen, P. K., Young, N. D., Nie, S., Ang, C. S., Williamson, N. A., Reid, G. E., \& Gasser, R. B. (2019). Dafachronic acid promotes larval development in Haemonchus contortus by modulating dauer signalling and lipid metabolism. PLoS Pathog, 15(7), e1007960. https://doi.org/10.1371/journal.ppat.1007960

Mahanti, P., Bose, N., Bethke, A., Judkins, J. C., Wollam, J., Dumas, K. J., Zimmerman, A. M., Campbell, S. L., Hu, P. J., Antebi, A., \& Schroeder, F. C. (2014). Comparative metabolomics reveals endogenous ligands of DAF-12, a nuclear hormone receptor, regulating C. elegans development and lifespan. Cell Metab, 19(1), 73-83. https://doi.org/10.1016/j.cmet.2013.11.024 
Milder, J. E., Walzer, P. D., Kilgore, G., Rutherford, I., \& Klein, M. (1981). Clinical features of Strongyloides stercoralis infection in an endemic area of the United States. Gastroenterology, 80(6), 1481-1488. https://www.ncbi.nlm.nih.gov/pubmed/7227772

Moloo, A. (2020, Dec., 17, 2020). A parasitic infection that can turn fatal with administration of corticosteroids. WHO News. https://www.who.int/news/item/17-12-2020-a-parasiticinfection-that-can-turn-fatal-with-administration-of-corticosteroids

Motola, D. L., Cummins, C. L., Rottiers, V., Sharma, K. K., Li, T., Li, Y., Suino-Powell, K., Xu, H. E., Auchus, R. J., Antebi, A., \& Mangelsdorf, D. J. (2006). Identification of ligands for DAF-12 that govern dauer formation and reproduction in C. elegans. Cell, 124(6), 1209-1223. https://doi.org/10.1016/j.cell.2006.01.037

NationalResearchCouncil. (2011). Guide for the Care and Use of Laboratory Animals: Eighth Edition. The National Academies Press. https://doi.org/doi:10.17226/12910

Nolan, T. J., Megyeri, Z., Bhopale, V. M., \& Schad, G. A. (1993). Strongyloides stercoralis: the first rodent model for uncomplicated and hyperinfective strongyloidiasis, the Mongolian gerbil (Meriones unguiculatus). J Infect Dis, 168(6), 1479-1484. https://doi.org/10.1093/infdis/168.6.1479

Ogawa, A., Streit, A., Antebi, A., \& Sommer, R. J. (2009). A conserved endocrine mechanism controls the formation of dauer and infective larvae in nematodes. Curr Biol, 19(1), 6771. https://doi.org/10.1016/j.cub.2008.11.063

Page, W., Judd, J. A., \& Bradbury, R. S. (2018). The unique life cycle of Strongyloides stercoralis and implications for public health action. Trop Med Infect Dis, 3(2). https://doi.org/10.3390/tropicalmed3020053

Patton, J. B., Bonne-Annee, S., Deckman, J., Hess, J. A., Torigian, A., Nolan, T. J., Wang, Z., Kliewer, S. A., Durham, A. C., Lee, J. J., Eberhard, M. L., Mangelsdorf, D. J., Lok, J. B., \& Abraham, D. (2018). Methylprednisolone acetate induces, and Delta7-dafachronic acid suppresses, Strongyloides stercoralis hyperinfection in NSG mice. Proc Natl Acad Sci U $S$ A, 115(1), 204-209. https://doi.org/10.1073/pnas.1712235114

Prichard, R. K. (2007). Ivermectin resistance and overview of the Consortium for Anthelmintic Resistance SNPs. Expert Opin Drug Discov, 2(s1), S41-52. https://doi.org/10.1517/17460441.2.S1.S41

Repetto, S. A., Ruybal, P., Batalla, E., Lopez, C., Fridman, V., Sierra, M., Radisic, M., Bravo, P. M., Risso, M. G., Gonzalez Cappa, S. M., \& Alba Soto, C. D. (2018). Strongyloidiasis Outside Endemic Areas: Long-term Parasitological and Clinical Follow-up After Ivermectin Treatment. Clin Infect Dis, 66(10), 1558-1565. https://doi.org/10.1093/cid/cix1069

Rottiers, V., Motola, D. L., Gerisch, B., Cummins, C. L., Nishiwaki, K., Mangelsdorf, D. J., \& Antebi, A. (2006). Hormonal control of C. elegans dauer formation and life span by a Rieske-like oxygenase. Dev Cell, 10(4), 473-482.

https://doi.org/10.1016/j.devcel.2006.02.008

Sharma, K. K., Wang, Z., Motola, D. L., Cummins, C. L., Mangelsdorf, D. J., \& Auchus, R. J. (2009). Synthesis and activity of dafachronic acid ligands for the C. elegans DAF-12 nuclear hormone receptor. Mol Endocrinol, 23(5), 640-648. https://doi.org/10.1210/me.2008-0415

Stoltzfus, J. D., Bart, S. M., \& Lok, J. B. (2014). cGMP and NHR signaling co-regulate expression of insulin-like peptides and developmental activation of infective larvae in 
Strongyloides stercoralis. PLoS Pathog, 10(7), e1004235. https://doi.org/10.1371/journal.ppat.1004235

Stoltzfus, J. D., Minot, S., Berriman, M., Nolan, T. J., \& Lok, J. B. (2012). RNAseq analysis of the parasitic nematode Strongyloides stercoralis reveals divergent regulation of canonical dauer pathways. PLoS Negl Trop Dis, 6(10), e1854. https://doi.org/10.1371/journal.pntd.0001854

Viney, M. E., \& Lok, J. B. (2015). The biology of Strongyloides spp. WormBook, 1-17. https://doi.org/10.1895/wormbook.1.141.2

Wang, Z., Schaffer, N. E., Kliewer, S. A., \& Mangelsdorf, D. J. (2017). Nuclear receptors: emerging drug targets for parasitic diseases. J Clin Invest, 127(4), 1165-1171. https://doi.org/10.1172/JCI88890

Wang, Z., Stoltzfus, J., You, Y. J., Ranjit, N., Tang, H., Xie, Y., Lok, J. B., Mangelsdorf, D. J., $\&$ Kliewer, S. A. (2015). The nuclear receptor DAF-12 regulates nutrient metabolism and reproductive growth in nematodes. PLoS Genet, 11(3), e1005027. https://doi.org/10.1371/journal.pgen.1005027

Wang, Z., Zhou, X. E., Motola, D. L., Gao, X., Suino-Powell, K., Conneely, A., Ogata, C., Sharma, K. K., Auchus, R. J., Lok, J. B., Hawdon, J. M., Kliewer, S. A., Xu, H. E., \& Mangelsdorf, D. J. (2009). Identification of the nuclear receptor DAF-12 as a therapeutic target in parasitic nematodes. Proc Natl Acad Sci U S A, 106(23), 9138-9143. https://doi.org/10.1073/pnas.0904064106

Wollam, J., Magner, D. B., Magomedova, L., Rass, E., Shen, Y., Rottiers, V., Habermann, B., Cummins, C. L., \& Antebi, A. (2012). A novel 3-hydroxysteroid dehydrogenase that regulates reproductive development and longevity. PLoS Biol, 10(4), e1001305. https://doi.org/10.1371/journal.pbio.1001305

Wollam, J., Magomedova, L., Magner, D. B., Shen, Y., Rottiers, V., Motola, D. L., Mangelsdorf, D. J., Cummins, C. L., \& Antebi, A. (2011). The Rieske oxygenase DAF-36 functions as a cholesterol 7-desaturase in steroidogenic pathways governing longevity. Aging Cell, 10(5), 879-884. https://doi.org/10.1111/j.1474-9726.2011.00733.x

Yamawaki, T. M., Berman, J. R., Suchanek-Kavipurapu, M., McCormick, M., Gaglia, M. M., Lee, S. J., \& Kenyon, C. (2010). The somatic reproductive tissues of $C$. elegans promote longevity through steroid hormone signaling. PLoS Biol, 8(8). https://doi.org/10.1371/journal.pbio.1000468

Zhao, L., Zhou, S., \& Gustafsson, J. A. (2019). Nuclear Receptors: Recent Drug Discovery for Cancer Therapies. Endocr Rev, 40(5), 1207-1249. https://doi.org/10.1210/er.2018-00222 


\section{ACKNOWLEDGMENTS}

1002

We thank members of the Mango/Kliewer lab and Dr. Jeffrey McDonald (UT

1003 Southwestern) for fruitful discussions. We thank Dr. Elissa Hallem (UCLA) for providing CRISPR

1004 plasmids. We thank Adeiye Pilgrim (Emory) for assistance with cloning. This work was supported

1005 by the National Institutes of Health (grant AI105856 to J.B.L., D.J.M. and S.A.K., R01GM141088

1006 to T.Q., and AI050886 to J.B.L), the Robert A. Welch Foundation (grants I-1275 to D.J.M., I-1558

1007 to S.A.K., and I-2010-20190330 to T.Q.), UT Southwestern Eugene McDermott Scholarship

1008 (T.Q.), and the Howard Hughes Medical Institute (D.J.M.).

\section{AUTHOR CONTRIBUTIONS}

1010 Z.W., J.B.L., S.A.K. and D.J.M. conceived and designed the study. J.B.L., S.A.K. and 1011 D.J.M. supervised the study. Z.W., M.C.C., J.D.C.S. and T.G.J. performed experiments. T.G.J and 1012 X.L. generated parasite material. J.T, H.D., and T.Q. optimized the synthesis and provided 1013 dafachronic acids. Z.W., J.B.L., S.A.K. and D.J.M. wrote the manuscript with contributions from 1014 all authors.

\section{DECLARATION OF INTERESTS}

The authors declare no competing interests. 